\title{
Multiwavelength follow-up of a rare IceCube neutrino multiplet
}

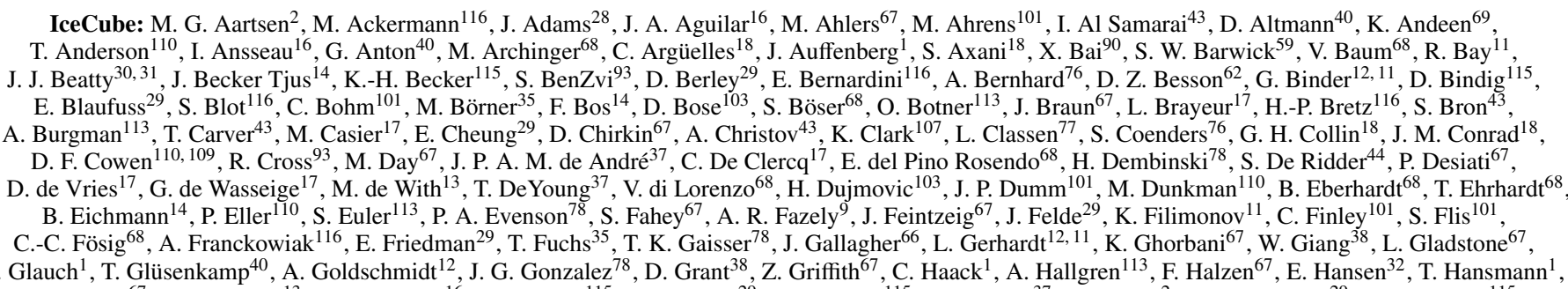
K. Hanson ${ }^{67}$, D. Hebecker ${ }^{13}$, D. Heereman ${ }^{16}$, K. Helbing ${ }^{115}$, R. Hellauer ${ }^{29}$, S. Hickford ${ }^{115}$, J. Hignight ${ }^{37}$, G. C. Hill ${ }^{2}$, K. D. Hoffman ${ }^{29}$, R. Hoffmann ${ }^{115}$, Hi K. Hoshina ${ }^{67}, 106$, F. Huang ${ }^{110}$, M. Huber ${ }^{76}$, K. Hultqvist ${ }^{101}$, S. In $^{103}$, A. Ishihara ${ }^{26}$, E. Jacobi ${ }^{116}$, G. S. Japaridze ${ }^{7}$, M. Jeong ${ }^{103}$, K. Jero ${ }^{67}$, B. J. P. Jones ${ }^{18}$,

W. Kang ${ }^{103}$, A. Kappes ${ }^{77}$, T. Karg ${ }^{116}$, A. Karle ${ }^{67}$, U. Katz ${ }^{40}$, M. Kauer ${ }^{67}$, A. Keivani ${ }^{110}$, J. L. Kelley ${ }^{67}$, A. Kheirandish ${ }^{67}$, J. Kim ${ }^{103}$, M. Kim ${ }^{103}$, T. Kintscher ${ }^{116}$, J. Kiryluk ${ }^{102}$, T. Kittler ${ }^{40}$, S. R. Klein ${ }^{12,11}$, G. Kohnen ${ }^{71}$, R. Koirala ${ }^{78}$, H. Kolanoski ${ }^{13}$, R. Konietz ${ }^{1}$, L. Köpke ${ }^{68}$, C. Kopper ${ }^{38}$, S. Kopper ${ }^{115}$, D. J. Koskinen ${ }^{32}$, M. Kowalski ${ }^{13,116}$, K. Krings $^{76}$, M. Kroll ${ }^{14}$, G. Krückl ${ }^{68}$, C. Krüger ${ }^{67}$, J. Kunnen ${ }^{17}$, S. Kunwar ${ }^{116}$, N. Kurahashi8 ${ }^{6}$, T. Kuwabara ${ }^{26}$, A. Kyriacou ${ }^{2}$, M. Labare $^{44}$,

J. L. Lanfranchi ${ }^{110}$, M. J. Larson ${ }^{32}$, F. Lauber ${ }^{115}$, M. Lesiak-Bzdak ${ }^{102}$, M. Leuermann ${ }^{1}$, L. Lu ${ }^{26}$, J. Lünemann ${ }^{17}$, J. Madsen ${ }^{92}$, G. Maggi ${ }^{17}$, K. B. M. Mahn ${ }^{37}$,

S. Mancina ${ }^{67}$, M. Mandelartz ${ }^{14}$, R. Maruyama ${ }^{79}$, K. Mase ${ }^{26}$, R. Maunu ${ }^{29}$, F. McNally ${ }^{67}$, K. Meagher ${ }^{16}$, M. Medici ${ }^{32}$, M. Meier ${ }^{35}$, T. Menne ${ }^{35}$, G. Merino ${ }^{67}$,

T. Meures ${ }^{16}$, S. Miarecki ${ }^{12,11}$, J. Micallef ${ }^{37}$, G. Momenté ${ }^{68}$, T. Montaruli $^{43}$, M. Moulai ${ }^{18}$, R. Nahnhauer ${ }^{116}$, U. Naumann ${ }^{115}$, G. Neer ${ }^{37}$, H. Niederhausen $^{102}$,

S. C. Nowicki ${ }^{38}$, D. R. Nygren ${ }^{12}$, A. Obertacke Pollmann ${ }^{115}$, A. Olivas ${ }^{29}$, A. O'Murchadha ${ }^{16}$, T. Palczewski ${ }^{12,11}$, H. Pandya $^{78}$, D. V. Pankova ${ }^{110}$, P. Peiffer ${ }^{68}$,

Ö. Penek ${ }^{1}$, J. A. Pepper ${ }^{108}$, C. Pérez de los $\operatorname{Heros}^{113}$, D. Pieloth ${ }^{35}$, E. Pinat ${ }^{16}$, P. B. Price ${ }^{11}$, G. T. Przybylski ${ }^{12}$, M. Quinnan ${ }^{110}$, C. Raab ${ }^{16}$, L. Rädel ${ }^{1}$, M. Rameez $^{32}$, K. Rawlins ${ }^{6}$, R. Reimann ${ }^{1}$, B. Relethford ${ }^{86}$, M. Relich ${ }^{26}$, E. Resconi ${ }^{76}$, W. Rhode ${ }^{35}$, M. Richman ${ }^{86}$, B. Riedel ${ }^{38}$, S. Robertson ${ }^{2}$, M. Rongen ${ }^{1}$, C. Rott ${ }^{103}$,

T. Ruhe ${ }^{35}$, D. Ryckbosch ${ }^{44}$, D. Rysewyk ${ }^{37}$, L. Sabbatini ${ }^{67}$, S. E. Sanchez Herrera ${ }^{38}$, A. Sandrock ${ }^{35}$, J. Sandroos ${ }^{68}$, S. Sarkar ${ }^{32,82}$, K. Satalecka ${ }^{116}$, P. Schlunder ${ }^{35}$, T. Schmidt ${ }^{29}$, S. Schoenen ${ }^{1}$, S. Schöneberg ${ }^{14}$, L. Schumacher ${ }^{1}$, D. Seckel ${ }^{78}$, S. Seunarine ${ }^{92}$, D. Soldin ${ }^{115}$, M. Song ${ }^{29}$, G. M. Spiczak ${ }^{92}$, C. Spiering ${ }^{116}$, J. Stachurska ${ }^{116}$, T. Stanev ${ }^{78}$, A. Stasik ${ }^{116}$, J. Stettner ${ }^{1}$, A. Steuer ${ }^{68}$, T. Stezelberger ${ }^{12}$, R. G. Stokstad ${ }^{12}$, A. Stöß126, R. Ström ${ }^{113}$, N. L. Strotjohann ${ }^{116, \star}$,

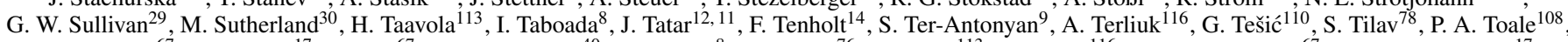
M. N. Tobin ${ }^{67}$, S. Toscano ${ }^{17}$, D. Tosi ${ }^{67}$, M. Tselengidou ${ }^{40}$, C. F. Tung ${ }^{8}$, A. Turcati ${ }^{76}$, E. Unger ${ }^{113}$, M. Usner ${ }^{116}$, J. Vandenbroucke ${ }^{67}$, N. van Eijndhoven ${ }^{17}$, S. Vanheule ${ }^{44}$, M. van Rossem ${ }^{67}$, J. van Santen ${ }^{116}$, M. Vehring ${ }^{1}$, M. Voge ${ }^{15}$, E. Vogel ${ }^{1}$, M. Vraeghe ${ }^{44}$, C. Walck ${ }^{101}$, A. Wallace ${ }^{2}$, M. Wallraff ${ }^{1}$, N. Wandkowsky ${ }^{67}$, A. Waza ${ }^{1}$, Ch. Weaver ${ }^{38}$, M. J. Weiss ${ }^{110}$, C. Wendt ${ }^{67}$, S. Westerhoff ${ }^{67}$, B. J. Whelan ${ }^{2}$, S. Wickmann ${ }^{1}$, K. Wiebe ${ }^{68}$, C. H. Wiebusch ${ }^{1}$, L. Wille ${ }^{67}$, D. R. Williams ${ }^{108}$, L. Wills ${ }^{86}$, M. Wolf ${ }^{101}$, T. R. Wood ${ }^{38}$, E. Woolsey ${ }^{38}$, K. Woschnagg ${ }^{11}$, D. L. Xu ${ }^{67}$, X. W. Xu ${ }^{9}$, Y. Xu ${ }^{102}$, J. P. Yanez ${ }^{38}$, G. Yodh ${ }^{59}$, S. Yoshida ${ }^{26}$, M. Zoll $^{101}$ ASAS-SN: K. Z. Stanek ${ }^{31,30}$, B. J. Shappee ${ }^{84,55}$, C. S. Kochanek ${ }^{31,30}$, T. W.-S. Holoien ${ }^{31,30}$, J. L. Prieto ${ }^{98,99}$

The Astrophysical Multimessenger Observatory Network: D. B. Fox ${ }^{109,111,112}$, J. J. DeLaunay ${ }^{110,111}$, C. F. Turley ${ }^{10,111}$, S. D. Barthelmy ${ }^{47}$, A. Y. Lien ${ }^{47}$, P. Mészáros ${ }^{110,109,111,112}$, K. Murase ${ }^{110,109,111,112}$

Fermi: D. Kocevski ${ }^{47}$, R. Buehler ${ }^{116}$, M. Giomi ${ }^{116}$, J. L. Racusin ${ }^{47}$

HAWC: A. Albert ${ }^{64}$, R. Alfaro ${ }^{20}$, C. Alvarez ${ }^{25}$, J. D. Álvarez ${ }^{73}$, R. Arceo $^{25}$, J. C. Arteaga-Velázquez ${ }^{73}$, H. A. Ayala Solares ${ }^{54}$, A. S. Barber ${ }^{94}$, N. Baustista-Elivar ${ }^{52}$, A. Becerril ${ }^{20}$, E. Belmont-Moreno ${ }^{20}$, A. Bernal ${ }^{19}$, C. Brisbois ${ }^{54}$, K. S. Caballero-Mora ${ }^{25}$, T. Capistrán ${ }^{88}$, A. Carramiñana ${ }^{88}$, S. Casanova ${ }^{61}$, M. Castillo ${ }^{73}$, U. Cottii ${ }^{73}$, S. Coutiño de León ${ }^{88}$, E. de la Fuente ${ }^{49}$, C. De León ${ }^{89}$, R. Diaz Hernandez ${ }^{88}$, J. C. Díaz-Vélez ${ }^{49,67}$, B. L. Dingus ${ }^{64}$, M. A. DuVernois ${ }^{67}$, R. W. Ellsworth ${ }^{41}$, K. Engel ${ }^{29}$, D. W. Fiorino ${ }^{29}$, N. Fraija ${ }^{19}$, J. A. García-González ${ }^{20}$, M. Gerhardt ${ }^{54}$, A. González Muñoz ${ }^{20}$, M. M. González ${ }^{19}$, J. A. Goodman ${ }^{29}$,

Z. Hampel-Arias ${ }^{67}$, J. P. Harding ${ }^{64}$, S. Hernandez ${ }^{20}$, C. M. Hui ${ }^{56}$, P. Hüntemeyer ${ }^{54}$, A. Iriarte ${ }^{19}$, A. Jardin-Blicq ${ }^{51}$, V. Joshi ${ }^{51}$, S. Kaufmannn ${ }^{25}$, A. Lara ${ }^{21}$,

R. J. Lauer ${ }^{3}$, W. H. Lee ${ }^{19}$, D. Lennarz ${ }^{7}$, H. León Vargas ${ }^{20}$, J. T. Linnemann ${ }^{37}$, G. Luis Raya ${ }^{52}$, R. Luna-García ${ }^{22}$, R. López-Coto ${ }^{51}$, K. Malone ${ }^{110}$,

S. S. Marinelli ${ }^{37}$, O. Martinez ${ }^{89}$, I. Martinez-Castellanos ${ }^{29}$, J. Martínez-Castro ${ }^{22}$, H. Martínez-Huerta ${ }^{23}$, J. A. Matthews ${ }^{3}$, P. Miranda-Romagnoli ${ }^{83}$, E. Moreno ${ }^{89}$, M. Mostafá ${ }^{110}$, L. Nellen ${ }^{24}$, M. Newbold ${ }^{94}$, M. U. Nisa ${ }^{93}$, R. Noriega-Papaqui ${ }^{83}$, R. Pelayo ${ }^{22}$, J. Pretz ${ }^{111}$, E. G. Pérez-Pérez ${ }^{52}$, Z. Ren ${ }^{3}$, C. D. Rho ${ }^{93}$, C. Rivière ${ }^{29}$,

D. Rosa-González ${ }^{88}$, M. Rosenberg ${ }^{111}$, F. Salesa Greus ${ }^{61}$, A. Sandoval ${ }^{20}$, M. Schneider ${ }^{97}$, H. Schoorlemmer ${ }^{51}$, G. Sinnis ${ }^{64}$, A. J. Smith ${ }^{29}$, R. W. Springer ${ }^{94}$, P. Surajbali ${ }^{51}$, O. Tibolla ${ }^{25}$, K. Tollefson ${ }^{37}$, I. Torres ${ }^{88}$, T. N. Ukwatta ${ }^{64}$, L. Villaseñor ${ }^{73}$, T. Weisgarber ${ }^{67}$, I. G. Wisher ${ }^{67}$, J. Wood ${ }^{67}$

$$
\text { T. Yapici }{ }^{37} \text {, A. Zepeda }{ }^{23}, \text { H. Zhou }{ }^{64}
$$

LCO: I. Arcavi ${ }^{45,96,95,39}$, G. Hosseinzadeh ${ }^{45,95}$, D. A. Howell ${ }^{45,95}$, S. Valenti ${ }^{34}$, C. McCully ${ }^{45,95}$

MASTER: V. M. Lipunov ${ }^{74,75}$, E. S. Gorbovskoy ${ }^{75}$, N. V. Tiurina ${ }^{75}$, P. V. Balanutsa ${ }^{75}$, A. S. Kuznetsov ${ }^{75}$, V. G. Kornilov ${ }^{74,75}$, V. Chazov ${ }^{75}$, N. M. Budnev ${ }^{58}$, O. A. Gress ${ }^{58}$, K. I. Ivanov ${ }^{58}$, A. G. Tlatov ${ }^{60}$, R. Rebolo Lopez ${ }^{105}$, M. Serra-Ricart ${ }^{105}$

Swift: P. A. Evans ${ }^{63}$, J. A. Kennea ${ }^{109}$, N. Gehrels ${ }^{47, \dagger}$, J. P. Osborne ${ }^{63}$, K. L. Page ${ }^{63}$

VERITAS: A. U. Abeysekara ${ }^{94}$, A. Archer ${ }^{100}$, W. Benbow ${ }^{4}$, R. Bird ${ }^{65}$, T. Brantseg 5 , V. Bugaev ${ }^{100}$, J. V Cardenzana ${ }^{5}$, M. P. Connolly ${ }^{42}$, W. Cui ${ }^{114,10}$, A. Falcone ${ }^{109}$, Q. Feng ${ }^{72}$, J. P. Finley ${ }^{114}$, H. Fleischhack ${ }^{116}$, L. Fortson ${ }^{70}$, A. Furniss ${ }^{50}$, S. Griffin ${ }^{72,100}$, J. Grube ${ }^{53}$, M. Hütten ${ }^{116}$, O. Hervet ${ }^{97}$, J. Holder ${ }^{78}$,

G. Hughes ${ }^{4}$, T. B. Humensky ${ }^{80}$, C. A. Johnson ${ }^{97}$, P. Kaaret5 ${ }^{77}$, P. Kar ${ }^{44}$, N. Kelley-Hoskins ${ }^{116}$, M. Kertzman ${ }^{48}$, M. Krause ${ }^{116}$, S. Kumar ${ }^{78}$, M. J. Lang ${ }^{42}$,

T. T. Y. Lin ${ }^{72}$, S. McArthur ${ }^{14}$, P. Moriarty ${ }^{42}$, R. Mukherjee ${ }^{81}$, D. Nieto ${ }^{80}$, R. A. Ong ${ }^{65}$, A. N. Otte ${ }^{8}$, M. Pohl ${ }^{87}, 1_{16}$, A. Popkow ${ }^{65}$, E. Pueschel ${ }^{36}$, J. Quinn ${ }^{36}$, K. Ragan ${ }^{72}$, P. T. Reynolds ${ }^{33}$, G. T. Richards ${ }^{8}$, E. Roache ${ }^{4}$, C. Rulten ${ }^{70}$, I. Sadeh ${ }^{116}$, M. Santander ${ }^{81}$, G. H. Sembroski ${ }^{114}$, D. Staszak ${ }^{27}$, S. Trépanier ${ }^{72}$, J. Tyler $^{72}$, S. P. Wakely ${ }^{27}$, A. Weinstein ${ }^{5}$, P. Wilcox ${ }^{57}$, A. Wilhelm ${ }^{87,116}$, D. A. Williams ${ }^{97}$, B. Zitzer ${ }^{72}$,

E. Bellm ${ }^{85}$, Z. Cano ${ }^{46}$, A. Gal-Yam ${ }^{91}$, D. A. Kann ${ }^{104}$, E. O. Ofek ${ }^{91}$, M. Rigault ${ }^{13}$, and M. Soumagnac ${ }^{91}$

\section{(Affiliations can be found after the references)}

Received 14 February 2017 / Accepted 30 July 2017

\footnotetext{
^ Corresponding author: N. L. Strotjohann, e-mail: nora.linn.strotjohann@desy.de
}

$\dagger$ Deceased: 6 Feb. 2017. 


\section{ABSTRACT}

On February 17, 2016, the IceCube real-time neutrino search identified, for the first time, three muon neutrino candidates arriving within $100 \mathrm{~s}$ of one another, consistent with coming from the same point in the sky. Such a triplet is expected once every 13.7 years as a random coincidence of background events. However, considering the lifetime of the follow-up program the probability of detecting at least one triplet from atmospheric background is $32 \%$. Follow-up observatories were notified in order to search for an electromagnetic counterpart. Observations were obtained by Swift's X-ray telescope, by ASAS-SN, LCO and MASTER at optical wavelengths, and by VERITAS in the very-high-energy gamma-ray regime. Moreover, the Swift BAT serendipitously observed the location $100 \mathrm{~s}$ after the first neutrino was detected, and data from the Fermi LAT and HAWC observatory were analyzed. We present details of the neutrino triplet and the follow-up observations. No likely electromagnetic counterpart was detected, and we discuss the implications of these constraints on candidate neutrino sources such as gamma-ray bursts, core-collapse supernovae and active galactic nucleus flares. This study illustrates the potential of and challenges for future follow-up campaigns.

Key words astroparticle physics - neutrinos - gamma-ray burst: general - supernovae: general - galaxies: active - X-rays: bursts

\section{Introduction}

In 2013, the IceCube neutrino observatory presented the first evidence for a high-energy flux of cosmic neutrinos (Aartsen et al. 2013, 2015a). While the evidence for their existence continues to mount, no explicit sources have been identified (see e.g., Aartsen et al. 2014, 2017b). The arrival directions of the events are distributed isotropically which likely implies that many events are of extragalactic origin.

High-energy neutrinos are produced when cosmic rays interact with ambient matter ( $\mathrm{pp}$ interactions) or photon fields ( $\mathrm{p} \gamma$ interactions). These interactions are expected to happen mainly within cosmic-ray sources where the target photon and/or matter densities are high. The detection of a neutrino source would imply that this source also accelerates cosmic rays.

Cosmic rays can be accelerated at collisionless shock fronts which are expected in a wide variety of astrophysical objects. Among those are gamma-ray bursts (GRBs; see e.g., Baerwald et al. 2015; Bustamante et al. 2015; Mészáros 2015), as well as the related class of low-luminosity GRBs (LLGRBs) or core-collapse supernovae (CCSNe) containing a choked jet (Murase \& Ioka 2013; Fraija 2014; Tamborra \& Ando 2016; Senno et al. 2016). CCSNe could in addition produce cosmic rays when their ejecta interact with circumstellar medium emitted by the star prior to the explosion (Murase et al. 2011, 2014; Katz et al. 2011). Other potential neutrino sources are active galactic nuclei (AGN; see Murase 2015, for a review), tidal disruption events (Farrar \& Piran 2014; Pfeffer et al. 2017; Wang \& Liu 2016) and starburst galaxies (Tamborra et al. 2014; Waxman 2015).

Thus far dedicated searches for correlations with specific source classes have not yielded a significant detection. At $90 \%$ confidence level, GRBs can at most account for $1 \%$ of the detected flux (Aartsen et al. 2015c) and the contribution from blazars has been limited to at most 30\% (Aartsen et al. 2017a). The non-detection of any neutrino sources implies that the astrophysical flux must originate from a large number of relatively faint neutrino sources (Ahlers \& Halzen 2014; Kowalski 2015; Murase \& Waxman 2016).

Several coincidences of neutrino events with astrophysical sources have been reported in the literature. For example a supernova of Type IIn was detected in follow-up observations of a neutrino doublet (Aartsen et al. 2015b). It is however likely unrelated given the large implied neutrino luminosity. Padovani et al. (2016) observe a correlation between extreme blazars and highenergy neutrino events and Kadler et al. (2016) found a bright gamma-ray outburst of a blazar which was aligned with a multi $\mathrm{PeV}$ neutrino event. However, all of these associations have a chance-coincidence probability of a few percent and are hence not significant detections.

The most energetic neutrino candidate detected so far, with a deposited energy of $2.6 \mathrm{PeV}$, was observed in June
2014 (Schoenen \& Raedel 2015; Aartsen et al. 2016a). The probability that this event was produced in the Earth's atmosphere is smaller than $1 \%$ and the angular uncertainty is $0.27^{\circ}$ (at $50 \%$ confidence) which makes it one of the best localized events observed with IceCube. However, no timely follow-up observations were triggered and a transient counterpart could have gone unnoticed. Since mid-2016, such events are identified, reconstructed, and published within minutes (Aartsen et al. 2017d) to allow quick follow-up observations (see Blaufuss 2016, as an example for the first published event).

In addition to the publicly announced high-energy neutrino alerts, IceCube has a real-time program that searches for multiple neutrinos from a similar direction (Abbasi et al. 2012b; Aartsen et al. 2017d). When two or more muon neutrino candidates are detected within $100 \mathrm{~s}$ of each other optical and X-ray observations can be triggered automatically (Evans et al. 2015; Aartsen et al. 2015b). Real-time follow-up observations are also triggered by the ANTARES neutrino telescope, but have not lead to the discovery of an electromagnetic counterpart (Ageron et al. 2012; Adrián-Martínez et al. 2016).

In February 2016, we found - for the first time - three events within this $100 \mathrm{~s}$ time window. The detection of such a triplet from atmospheric background is not unlikely considering that the search has been running since December 2008 (compare Sect. 3.2). However, since it is the most significant neutrino multiplet detected so far, multiwavelength follow-up observations were triggered to search for a potential electromagnetic counterpart.

In this paper we present details of the neutrino triplet and results of the follow-up observations. In Sect. 2 we introduce the follow-up program. The properties of the triplet are given in Sect. 3. The follow-up observations, covering optical wavelengths up to very-high-energy (VHE) gamma rays, are presented in Sect. 4. Finally, in Sect. 5 we draw conclusions from the various observations and discuss the sensitivity of our program to candidate neutrino source classes.

\section{The IceCube follow-up program}

\subsection{The IceCube neutrino telescope}

IceCube is a cubic-kilometer-sized neutrino detector installed in the ice at the geographic South Pole between a depth of $1450 \mathrm{~m}$ and $2450 \mathrm{~m}$ (Aartsen et al. 2017c). An array of 5160 digital optical modules (DOMs; Abbasi et al. 2009, 2010a), which are deployed in the ice, detects the Cherenkov radiation from secondary particles produced in neutrino interactions (Achterberg et al. 2006). Based on the pattern of the Cherenkov light, both the direction and energy of the neutrinos can be measured. The detector has been running in its full configuration since May 2011.

Neutrinos can interact and produce secondary particles through neutral current (NC) interactions or through charged 
current (CC) interactions. $\mathrm{CC}$ interactions induced by electron or tau neutrinos, as well as NC interactions induced by any neutrino flavor, produce localized, almost spherical light patterns inside the detector (see Aartsen et al. 2013, for example), which makes directional reconstructions challenging. Muons produced in $v_{\mu}$ CC interactions, on the other hand, can travel up to several kilometers in the ice and emit Cherenkov light along their trajectories. These events are called tracks and their source directions can be reconstructed to better than one degree if their energy is $>1 \mathrm{TeV}$ (Aartsen et al. 2017b). Track events often extend beyond the detector volume which means that the detected energy is a lower limit on the neutrino energy. Due to their superior angular resolution, track events are preferred for neutrino astronomy and the real-time system only uses $v_{\mu} \mathrm{CC}$ events.

\subsection{Real-time event selection}

IceCube has several real-time follow-up programs which select events and generate alerts in different ways (Aartsen et al. 2017d). The neutrino alert described in this paper was found by the optical follow-up program (see also Abbasi et al. 2012b; Evans et al. 2015; Aartsen et al. 2015b) which searches for short transient neutrino sources and triggers optical telescopes as well as the Swift X-ray telescope.

Event selection starts from the online Muon Filter selection that identifies high-quality muon tracks with a rate of about $40 \mathrm{~Hz}$. This rate is dominated by muons produced in cosmicray air showers. To increase the neutrino purity of the sample, more advanced and time-consuming reconstructions are required. Since computing power at the South Pole is limited, these reconstructions can only be applied to a subset of events. At the South Pole, the Online Level 2 Filter uses the outcome of a maximum likelihood reconstruction to further reduce contamination from atmospheric muons. This reconstruction takes into account how photons propagate to the optical modules in the detector. Selection criteria are, for example, the quality of the likelihood fit and the total number of modules that detected a photon. After application of these criteria, the event rate is reduced to $5 \mathrm{~Hz}$, which is low enough to apply more sophisticated and timeconsuming reconstruction algorithms (see Aartsen et al. 2015b, for a more detailed description). Based on the results of these reconstructions, the most signal-like events are selected using a multivariate classifier (see Aartsen et al. 2017d, for more details on the event selection and data transmission).

To avoid the background of atmospheric muons entering the detector from above, the follow-up program only uses events coming from below and is hence only sensitive to sources in the Northern sky. The final event rate is $3 \mathrm{mHz}$ and has a neutrino purity of $\sim 80 \%$. Most selected neutrino candidates are produced in atmospheric showers and out of $\sim 10^{5}$ detected events per year only several hundreds are expected to be of cosmic origin (see Sect. 5.1). To overcome this background we restrict our search to short transient sources which are detected with several neutrinos.

\subsection{Alert generation}

The IceCube optical follow-up program has been running since December 2008 (Abbasi et al. 2012b). After selecting a stream dominated by upward-going neutrino events, it searches for coincident events. A multiplet alert is generated whenever two or more tracks arrive within $100 \mathrm{~s}$ with an angular separation of less than $3.5^{\circ}$. The length of the time window was chosen such

\footnotetext{
1 While IceCube was running in the 40 and 59 string configuration the required angular separation was $4^{\circ}$ (2008-12-16 to 2009-12-31).
}

that it covers the typical duration of a SN core-collapse and the lifetime of a jet in a GRB (compare Abbasi et al. 2012b). To measure the significance of a neutrino doublet, a quality parameter is calculated using Eq. (1) in Aartsen et al. (2015b). Based on this parameter, we select the doublets that are the least likely to be chance coincidences of background events (i.e., the reconstructed directions of the two events are consistent within the errors, they are detected within a short time, and both events are well localized). Follow-up observations are triggered automatically for doublets above a fixed significance threshold. Multiplets consisting of more than two events are rare (compare Sect. 3.2) and no additional significance cut is applied.

We use simulated neutrino events following an $E^{-2.5}$ spectrum to quantify the efficiency of the multiplet selection process. If three neutrinos from a transient source pass the event selection within less than $100 \mathrm{~s}$, a triplet or two doublets with one common event are detected in $79 \%$ of the cases. One doublet would be detected if one of the three events was separated by more than $3.5^{\circ}$ from the two other events, which happens with a probability of $18 \%$. There is a $3 \%$ chance that the reconstructed directions of all three neutrinos would be separated by more than $3.5^{\circ}$ and no alert would be issued.

\section{The alert}

Two neutrino doublets, which have one event in common, were found on 2016-02-17 19:21:31.65 (detection time of the first neutrino event, referred to as T0 in the following; all dates are in UTC). All three events arrived within less than $100 \mathrm{~s}$. They were not automatically identified as a triplet because the second and third events were separated by $3.6^{\circ}$, while our cut is at an angular distance of $3.5^{\circ}$. However, for convenience we refer to the alert as a triplet in the following.

Neither doublet passed the required significance cut for individual doublets to be automatically forwarded to the Palomar Transient Factory (PTF; Law et al. 2009; Rau et al. 2009) or to the Swift satellite (Gehrels et al. 2004). More details on the individual events are given in Table 1 and the projection of the events on the sky is shown in Fig. 1.

The combined average neutrino direction is $\mathrm{RA}=26.1^{\circ}$ and Dec $=39.5^{\circ} \mathrm{J} 2000$ with a $50 \%$ error circle of $1.0^{\circ}$ and a $90 \%$ error circle of $3.6^{\circ}$. This direction corresponds to the weighted arithmetic mean position taking into account the angular uncertainties of the individual events, $\sigma_{i}$. The error on the combined direction is defined as $\sigma_{w}=\left(\sum_{i=1}^{N} \sigma_{i}^{-2}\right)^{-1 / 2}$, where $N=3$ is the number of events. To estimate the $90 \%$ error circle of the detected events we use simulated neutrino events which deposited a similar amount of energy in the detector. We determine by what factor the $50 \%$ error circle has to be increased such that it contains the true neutrino direction for $90 \%$ of the simulated events.

All quoted directions were obtained with the multiphotoelectron (MPE) fit (see Ahrens et al. 2004) which was used for the follow-up program at the time of the alert. An improved version of this algorithm, called Spline MPE, uses a more realistic model of light propagation in ice and on average reaches a more precise reconstruction of the direction (Aartsen et al. 2014). The Spline MPE reconstruction has been used for the follow-up program since May 2016. The Spline MPE fit yields shifted coordinates which are shown in brackets in Table 1. The reconstructed direction changes the most for the first event, which deposited light in a relatively small number of DOMs due to its low energy. Based on the Spline MPE fit, the average direction of all three events is $\mathrm{RA}=25.7^{\circ}, \mathrm{Dec}=39.6^{\circ}$ with error circles of $0.6^{\circ}(50 \%)$ and $1.9^{\circ}(90 \%)$. 
Table 1. Details on IceCube events.

\begin{tabular}{llllllll}
\hline \hline ID & IceCube event ID & Alert ID & $\begin{array}{l}\text { Time } \\
(\mathrm{s})\end{array}$ & $\begin{array}{l}\text { RA } \\
\left({ }^{\circ}\right)\end{array}$ & $\begin{array}{l}\text { Dec } \\
\left({ }^{\circ}\right)\end{array}$ & $\begin{array}{l}\text { Error } \\
\left({ }^{\circ}\right)\end{array}$ & $\begin{array}{l}\text { Deposited energy } \\
(\mathrm{TeV})\end{array}$ \\
\hline 1 & 62474825 & 7,8 & 0 & $26.0[30.2]$ & $39.9[43.2]$ & $4.5[3.6]$ & 0.26 \\
2 & 62636100 & 7 & +55.4 & $24.4[24.2]$ & $37.8[38.4]$ & $1.6[0.9]$ & 1.1 \\
3 & 62729180 & 8 & +87.3 & $27.2[26.8]$ & $40.7[40.7]$ & $1.4[0.9]$ & 0.52 \\
\hline
\end{tabular}

Notes. The directions are the result of the reconstruction algorithm that was used in the follow-up program at the time of the alert (MPE fit), while the values in brackets result from an alternative reconstruction algorithm with an improved ice model (Spline MPE fit). The error on the direction is the radius of the 50\% error circle. The last column shows an estimate of the energy deposited by the muons in the detector, which is a lower limit on the neutrino energy. All times are relative to 2016-02-17 19:21:31.65 UTC.

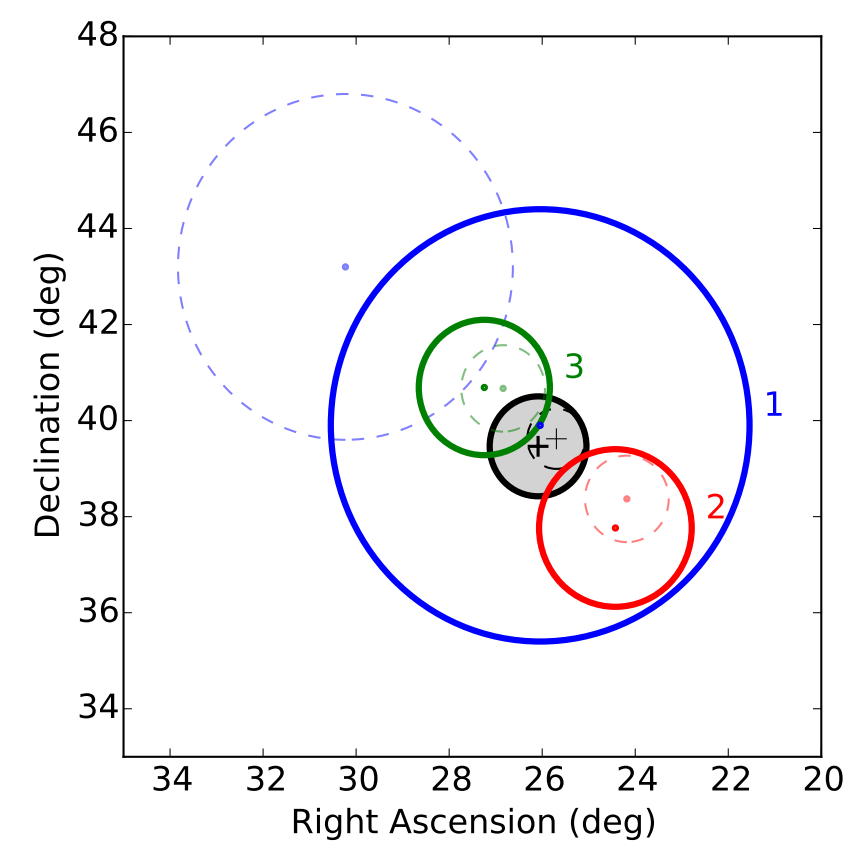

Fig. 1. Location of the three neutrino candidates in the triplet with their $50 \%$ error circles. The plus sign shows the combined direction and the shaded circle is the combined $50 \%$ error circle. The solid circles show the results of the MPE reconstruction and the thin dashed circles correspond to the results of the Spline MPE reconstruction (compare Table 1). All further results are based on the MPE reconstruction which was the reconstruction used for the follow-up program until May 2016.

Based on the Spline MPE reconstruction, events 1 and 2 (see Table 1) would no longer form a doublet, while events 2 and 3 would have formed a doublet. We expect the detection of 66 doublets per year due to background, and the $\sim 5$ most significant doublets are followed up (see Sect. 2.3). The doublet consisting of events 2 and 3 does not pass the significance threshold (compare Sect. 2.3). Hence, the alert would not have been considered interesting and no follow-up observations would have been triggered even if our program had been running with the Spline MPE reconstruction at the time of the alert.

We used simulated neutrino events following an $E^{-2.5}$ neutrino spectrum (compare Sect. 5.1) to calculate the probability that three events from a point source form a triplet based on the MPE reconstruction, which is not recovered when using the Spline MPE algorithm. The resulting probability is $8 \%$. For background triplets (i.e., events that are aligned by chance but do not stem from a point source) we evaluate scrambled data (compare Sect. 3.2) and find that the probability is 36\%. The fact that the triplet is not re-detected when using the Spline MPE algorithm is therefore a slight indication that it might not be of astrophysical origin, but a coincidence of aligned background events.

To test more precisely whether the three events are consistent with a single point source origin we simulated events from a similar zenith range. The true direction of the events is shifted to the same position and we select events with comparable estimated angular errors. We then check how often they are reconstructed further from their true direction than the three detected events. We quantify this by defining a test statistic equivalent to the spatial term used in the standard point source analysis (Eq. (3) in Aartsen et al. 2017b) and find that this happens in $\sim 75 \%$ ( $\sim 50 \%$ using the SplineMPE results) of all cases. Therefore, the detected events are consistent with a point source origin when considering their errors and the detector properties for this zenith direction.

All following analyses are based on the MPE position and error estimate which are shown as solid lines in Fig. 1. Compared to the angular separations between the neutrino candidates the mean position only changes slightly and the $50 \%$ error circle of the MPE reconstruction fully contains the $50 \%$ error circle of the Spline MPE fit.

\subsection{Detector stability}

Before triggering follow-up observations we examined the status of the detector carefully. A set of selected trigger and filter rates related to the analysis are monitored in real-time. Figure 2 shows the rate of the Simple Multiplicity Trigger, the Muon Filter, and the Online Level 2 Filter (see Sect. 2.2) near the time of the events. A Simple Multiplicity consists of eight DOMs forming at least four pairs in close temporal and spatial coincidence which trigger within $5 \mu \mathrm{s}$.

These quantities are sensitive to disturbances in the datacollection process (Aartsen et al. 2017d). These disturbances are classified as either internal, such as interrupted connections to a segment of the detector, or external, such as interference from other experiments at the South Pole. Periods of bad operating conditions can be flagged by monitoring the moving average of the rates and comparing it to expected statistical fluctuations. This system has operated for several years and has reliably identified occasional internal and external disturbances during that period. No significant deviation from normal detector behavior was observed for a time period spanning several hours around the events in the triplet.

In addition we generated test alerts which consisted of two events within $100 \mathrm{~s}$ that are separated by more than $3.5^{\circ}$, but less than $7.5^{\circ}$. The test alert rate did not show any anomalies around the time of the alert. We hence conclude that the detector was stable when the neutrino triplet was detected. 
IceCube et al.: Follow-up of a neutrino multiplet

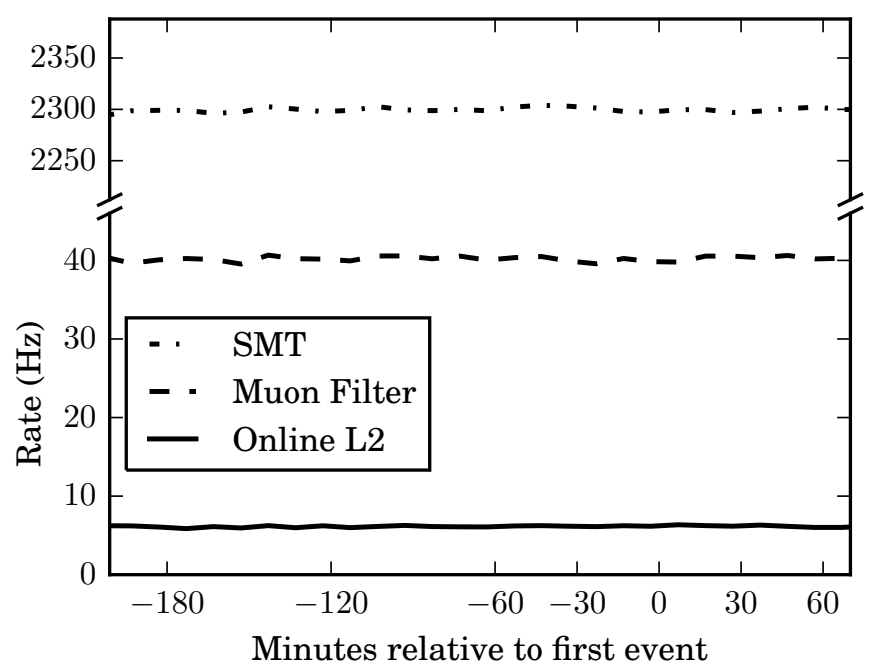

Fig. 2. Temporal behavior of different filter rates: the Simple Multiplicity Trigger, Muon Filter, and Online Level 2 rate. No significant deviation from normal detector behavior was observed around the time of the alert.

\subsection{Significance calculation}

To quantify the significance of the neutrino detection, we calculate how often triplets are expected from chance coincidences of background events. We use the data obtained during the previous IceCube season from 2014-05-06 to 2015-05-18 when the follow-up program was running in the same configuration. Considering only the time when the follow-up program was running stably, the uptime of this season was 359 days, during which 100799 neutrino candidates passed the event selection of the follow-up program.

To estimate the multiplet false positive rate from atmospheric backgrounds, we randomly exchanged the detection times of all events during this data-taking season. The event directions in detector coordinates remained the same, but the equatorial coordinates were recalculated using the newly assigned detection time. This method preserves both the temporal variations in the data (e.g., seasonal variations; see Abbasi et al. 2010b) and directional effects caused by the detector geometry. At the same time, any potential signal from a transient or steady source is smeared out.

To the generated background data, we applied our a priori cuts and searched for neutrino doublets (two events arriving within $100 \mathrm{~s}$ and with an angular separation of at most $3.5^{\circ}$ ). We then counted how many doublets had at least one neutrino event in common and found that such overlapping doublets or triplets are expected $0.0732 \pm 0.0009$ times per full year of live time, hence one every $13.7 \mathrm{yr}$ assuming the configuration in which the program was running at the time of the alert ${ }^{2}$. The expected number of background alerts is calculated for every season since the start of the follow-up program in December 2008. Within this time both the event selection and alert generation of the follow-up program were improved yielding different sensitivities. Moreover, we consider the down time of the follow-up program. Adding up the different contributions since 2008, the total number of expected triplets from background was 0.38 at the arrival time of the first triplet. The probability to detect one or

\footnotetext{
2 We emphasize that our definition of a triplet only requires that one of the three events forms a doublet with the two other ones. The two other events can therefore be separated by more than $3.5^{\circ}$ and do not have to arrive within $100 \mathrm{~s}$.
}

more triplets from background is hence $32 \%$. The detected neutrino triplet may therefore be caused by a chance alignment of background events.

\section{Follow-up observations}

The neutrino triplet was not automatically forwarded to any follow-up observatory because it did not pass the required criteria (all events within $3.5^{\circ}$ ) and neither of the individual doublets reached the required significance threshold for triggering followup observations. As calculated in Sect. 3.2 the detection of a triplet from background is expected once every $13.7 \mathrm{yr}$, which makes it a rare alert and the most significant neutrino multiplet detected so far. Therefore, the IceCube Collaboration decided to notify the partners providing electromagnetic follow-up observations. Our follow-up partners were informed $22 \mathrm{~h}$ after the detection of the triplet. In case of automatic forwarding, the median latency for triggering follow-up observatories is $\sim 1 \mathrm{~min}$.

The triplet direction was $\sim 70^{\circ}$ from the Sun and difficult to observe from ground-based observatories since it was located close to the horizon during night time and a large air mass impaired the image quality.

Several source classes have been suggested as potential transient neutrino sources. We therefore obtained multiwavelength observations at different times after the neutrino detection. We specifically search for GRBs, CCSNe (which might contain choked jets) and AGN flares. In this section we present reports on the observations obtained with optical (Sect. 4.1), X-ray (Sect. 4.2) and gamma-ray (Sect. 4.3) telescopes. The results are summarized and evaluated in Sect. 5.

\subsection{Optical observations}

Optical follow-up observations were obtained with ASAS-SN, MASTER, and LCO. No observations could be obtained with the PTF P48 telescope which was undergoing engineering work. In addition to these follow-up observations, we also analyze archival data obtained within a period of 30 days before the neutrino triplet.

\subsubsection{All-Sky Automated Survey for SuperNovae}

The All-Sky Automated Survey for SuperNovae (ASAS-SN or "Assassin"; Shappee et al. 2014) monitors the whole sky down to a limiting magnitude of $V \sim 17 \mathrm{mag}$. The focus of the survey is to find nearby supernovae ( $\mathrm{SNe}$ ) and other bright transient sources. Currently, ASAS-SN consists of two fully robotic units with four $14 \mathrm{~cm}$ telescopes each on Mount Haleakala in Hawaii and Cerro Tololo in Chile. These eight telescopes allow ASAS-SN to survey $20000 \mathrm{deg}^{2}$ per night, covering the entire visible sky every two days. The pipeline is fully automatic and discoveries are announced within hours of the data being collected. The data are photometrically calibrated using the AAVSO Photometric All-Sky Survey (APASS; Henden et al. 2015).

The ASAS-SN "Brutus" station in Hawaii has regularly observed the field containing the triplet position since 2013-10-27, obtaining 408 ninety-second $V$-band images on 178 separate nights. Before the neutrino trigger, this field was last observed two weeks earlier, on 2016-02-03, as the observability of this field was limited due to the Sun angle. In Table B.1 we list the dates on which this field was observed during the 30 days before the trigger, and also the typical $5 \sigma \mathrm{V}$ band 
detection limit reached, in the $3 \times 90$-s dithered exposures. The resulting limits are shown in Sect. 5.2.

Following the neutrino trigger, we scheduled $20 \times 90 \mathrm{~s}$ exposures of the field containing the trigger position, which were taken between UTC 2016-02-19.229 and 2016-02-19.253, that is, $34 \mathrm{~h}$ after the neutrino detection. The ASAS-SN field contains about $90 \%$ of the final $50 \%$ error circle of $1^{\circ}$. Because of the bright Moon, the combined depth of $V \lesssim 18.0$ is relatively shallow while the $5 \sigma$ depth of the individual 90 -s exposures is $V \lesssim 16.5$. No transient sources were detected.

\subsubsection{Las Cumbres Observatory}

The Las Cumbres Observatory ( $\mathrm{LCO}^{3}$; Brown et al. 2013) consists of seven $0.4 \mathrm{~m}$, nine $1 \mathrm{~m}$ and two $2 \mathrm{~m}$ robotic telescopes situated in six sites around the world (two additional $1 \mathrm{~m}$ telescopes will be deployed in the near future to a seventh site). The network specializes in time domain astronomy, and has the capability of performing immediate target-of-opportunity observations of almost any point in the sky within minutes.

The error circle of the neutrino triplet was tiled with nine pointings that were observed with the LCO $1 \mathrm{~m}$ telescope at the McDonald observatory in Texas. The observations cover the inner $\sim 60 \%$ of the $50 \%$ error circle of the final triplet location. Observations started $30 \mathrm{~h}$ after the neutrino detection and various combinations of UBVgri filters were used on different nights (Table B.2 and Sect. 5.2). The limiting magnitudes were calculated following calibration to the APASS catalog (see Appendix B of Valenti et al. 2016, for more details). Due to the proximity of the field to the sun, additional epochs could not be obtained in the weeks following the alert to determine whether or not any transient sources were present in the images.

\subsubsection{Mobile Astronomical System of the Telescope-Robots}

The Mobile Astronomical System of the Telescope-Robots (MASTER; Lipunov et al. 2010; Kornilov et al. 2012; Gorbovskoy et al. 2013) Global Robotic Net consists of seven observatories in both hemispheres (see Table 2). All MASTER observatories include identical twin $40 \mathrm{~cm}$ wide-field telescopes with two 4 square degree FoV which monitor the sky down to 21 st magnitude. In divergent mode, the twin telescopes can cover 8 square degrees per exposure and the telescope mounts allow rapid pointing to follow up short transient sources. Each MASTER node is equipped with BVRI Johnson/Bessel filters, two orthogonal polarization filters and two white filters (called unfiltered). To collect as many photons as possible, the MASTER telescopes are usually operated without a filter when searching for transients. In addition, each observatory hosts very-wide-field cameras which cover 400 square degrees and are sensitive to sources brighter than 15th magnitude.

An important component of MASTER is its in-house detection software which provides photometric and astrometric information about all optical sources in the image within 1-2 min of the frame readout. The processing time includes primary reduction (bias, dark, flat field), source extraction with help of the SExtractor algorithm ${ }^{4}$ (Bertin \& Arnouts 1996), the identification of cataloged objects and the selection of unknown objects. New sources detected in two images at the same position are classified as optical transients (Lipunov et al. 2016). The unfiltered magnitudes are calibrated using stars from the USNO-B1

\footnotetext{
3 http://lco.global

4 http://www.astromatic.net/software/sextractor
}

catalog where the catalog magnitudes are converted to unfiltered magnitudes via $0.2 \times B+0.8 \times R$. For each image, a limiting magnitude is calculated.

The MASTER network received the neutrino triplet coordinates by email at 2016-02-18 17:15:58 UTC. The altitudes and visibility constraints of the position at the different observatories are listed in Table 2 for the time when the neutrino detection was communicated. Observations started at the MASTERKislovodsk telescopes within less than one hour and the position was monitored by MASTER-Kislovodsk, MASTER-Tunka, and MASTER-IAC for the following month (compare Table B.1).

The majority of the observations listed in Table B.1 are centered on the triplet position and include the complete $50 \%$ error circle of the final position. Moreover, except for small gaps, the complete $90 \%$ error circle was covered both before and after the neutrino detection. No transients were found above the $5 \sigma$ limiting magnitudes given in Table B. 1 and shown in Sect. 5.2. The very- wide-field cameras did not detect any transient brighter than 15 th magnitude within the 400 square degrees surrounding the triplet location.

\subsection{X-ray observations}

We triggered the X-Ray Telescope (XRT) on board the Swift satellite (Gehrels et al. 2004) to search for GRB afterglows, AGN flares, or other X-ray transients (see Sect. 4.2.2). By chance, the Swift Burst Alert Telescope (BAT; Barthelmy et al. 2005) observed the triplet position within a minute after the neutrino detection as described in Sect. 4.2.1.

\subsubsection{Swift Burst Alert Telescope}

Swift BAT detects hard X-rays in the energy range from 15 to $150 \mathrm{keV}$. The FoV covers about $10 \%$ of the sky and the detector is illuminated through a partially coded aperture mask.

Just $100 \mathrm{~s}$ after the first neutrino was detected, the Swift satellite completed a preplanned slew to $\mathrm{RA}=23.38^{\circ}$, Dec $=$ $+41.12^{\circ}$ which placed the triplet position within the BAT FoV, at a partial coding fraction of $60 \%$. We retrieved the BAT data for this pointing from the Swift Quick Look website (ObsID 00085146016). No rate- or image-triggered transients were detected above the significance threshold of $\mathcal{S}>6.5 \sigma$ during the pointing, so only survey mode data are available. Survey data for the pointing consist of three exposures of $59 \mathrm{~s}, 10 \mathrm{~s}$, and $15 \mathrm{~s}$, with intervening gaps for maintenance operations. The BAT analysis was conducted using the HEASOFT ${ }^{5}$ (v. 6.18) software tools and calibration, closely following the analyses from Markwardt et al. (2005), Tueller et al. (2008, 2010) and Baumgartner et al. (2013).

We used the HEASOFT tool batcelldetect on the summed exposure as well as on the first exposure over the full bandpass (15-150 keV), with a detection threshold of $\mathcal{S}=3.5 \sigma$ (the lowest allowed setting). The most significant detection within the triplet $90 \%$ confidence region was in the first exposure at $\mathrm{RA}=28.6083^{\circ}, \mathrm{Dec}=37.34583^{\circ}$ (henceforth referred to as the BAT Blip) with single-trial significance $\mathcal{S}=4.6 \sigma$.

To estimate the significance of the BAT Blip given the search area, we find the number of similar or more significant fluctuations in a rectangular region of the BAT image plane centered around the position of BAT Blip in 2655 BAT pointings with similar exposure times. We find an average of 0.13 such candidate sources per pointing. Since the triplet $90 \%$-confidence

\footnotetext{
5 HEASOFT website: http://heasarc.nasa.gov/lheasoft/
} 
IceCube et al.: Follow-up of a neutrino multiplet

Table 2. Observing conditions at the MASTER telescopes at the time 2016-02-18 17:15:58 UTC.

\begin{tabular}{llll}
\hline \hline MASTER node & $\begin{array}{l}\text { Object altitude } \\
\left({ }^{\circ}\right)\end{array}$ & $\begin{array}{l}\text { Sun altitude } \\
\left({ }^{\circ}\right)\end{array}$ & Notes \\
\hline MASTER-Amur & 3.98 & -47.01 & Too close to the horizon for good observations \\
MASTER-Tunka & 13.45 & -49.91 & Cloudy and too close to the horizon for good observations \\
MASTER-Ural & 37.06 & -33.25 & Bad weather \\
MASTER-Kislovodsk & 43.50 & -28.31 & Good conditions, observations began \\
MASTER-SAAO & -8.36 & 0.93 & Below the horizon at night time \\
MASTER-IAC & 78.22 & 20.25 & Snow storm \\
MASTER-OAFA & -1.1 & 69.06 & Below the horizon at night time \\
\hline
\end{tabular}

region corresponds to $41 \%$ of the rectangular region, this yields a $p$-value of $p=9.9 \%$ for the BAT Blip. A trial factor penalty of two was included since both the summed and the first exposure were analyzed. The BAT Blip is hence consistent with a random fluctuation of the background.

Flux upper limits were derived from the summed exposure noise map, including the BAT Blip, over the triplet $90 \%$ confidence region, and we find a $4 \sigma$ upper limit to the fluence of $3.3 \times 10^{-7} \mathrm{erg} \mathrm{cm}^{-2}$ for the energy range of $15-150 \mathrm{keV}$. This corresponds to a limit of $3.9 \times 10^{-9} \mathrm{erg} \mathrm{cm}^{-2} \mathrm{~s}^{-1}$ on the average flux between $100 \mathrm{~s}$ and $256 \mathrm{~s}$ after the detection of the first neutrino. BAT count limits are converted to fluences using the PIMMS $^{6}$ online tool, assuming a power law with a spectral index of $\Gamma=-2$. This spectral index corresponds to a typical GRB spectrum in this energy range. It is moreover very close to the mean AGN spectral index which was measured to be -1.95 by Burlon et al. (2011). In Sect. 5.3 we compare the limit to typical prompt fluxes of GRBs detected by the BAT.

\subsubsection{Swift X-Ray Telescope}

The Swift XRT is an X-ray imaging spectrometer sensitive to the energy range $0.3-10 \mathrm{keV}$. The telescope's FoV has a diameter of $0.4^{\circ}$. To search for possible X-ray counterparts to the neutrino triplet over the largest feasible region, we requested a 37pointing mosaic of Swift observations. These observations began at 2016-02-18 17:57:42 (22.6 h after the neutrino detection; Target IDs 34342 to 34379), with the resulting exposure map shown in Fig. 3. The achieved exposure per pointing is $0.3-0.4 \mathrm{ks}$. Data were analyzed as described in Evans et al. (2015), leading to a single unified X-ray image, exposure map, and list of X-ray sources. The Swift XRT observations cover nearly the complete $50 \%$ containment region.

Six X-ray sources were identified (Table 3) with the detection flag good which means that their probability of being spurious is $<0.3 \%$ (Evans et al. 2015). As revealed from searches of the NASA Extragalactic Database ${ }^{7}$ and examination of archival optical images, $\mathrm{X} 1$ is spacially coincident with a known Seyfert 1 galaxy; X2, X3, X4, and X5 correspond to cataloged stars and $\mathrm{X} 6$ remains unidentified. We note that X-rays associated with a bright star were detected when Swift followed up a neutrino candidate detected by the ANTARES neutrino telescope (Dornic et al. 2015; Smartt et al. 2015). The large number of stars detected in our observations shows that such chance coincidences are frequent. We do not consider the stars as potential sources of high-energy neutrinos.

\footnotetext{
6 Available at https://heasarc.gsfc.nasa.gov/docs/ software/tools/pimms.html

7 NASA Extragalactic Database: https://ned.ipac.caltech. edu/
}

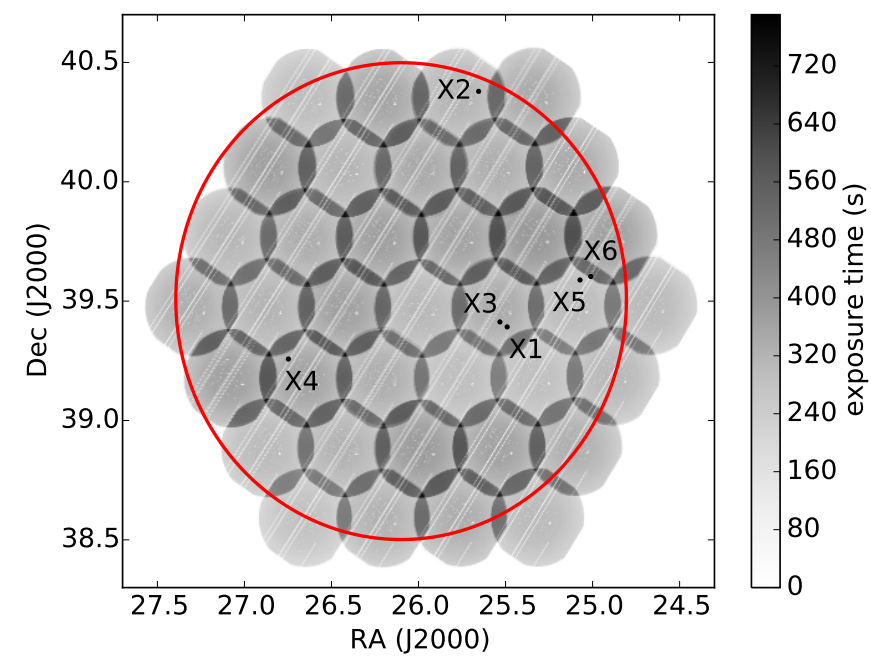

Fig. 3. Exposure map of the 37 Swift XRT pointings averaging $320 \mathrm{~s}$ per tiling. The red circle shows the $50 \%$ confidence bound to the triplet position. XRT sources (compare Table 3) are shown as black points.

The X-ray source X1 is classified as a Flat Spectrum Radio Quasar by Healey et al. (2007) and is located at a redshift of $z=0.08$ (Wills \& Browne 1986); it has been detected several times by ROSAT, XMM-Newton and the Swift XRT. Compared to the previous detections, X1 was not flaring during these XRT observations.

Among the identified sources, X6 is unique in not having an obvious counterpart within its $90 \%$ error circle. To refine the localization and study the X-ray variability, X6 was followed up with $1 \mathrm{ks}$ and $8.6 \mathrm{ks}$ Swift observations on 2016-03-18 and 2016-07-23 (Target ID 34429). The source was re-detected in the deepest XRT observation; it faded by a factor of nine within five months. The XRT light curve, shown in Fig. A.1, is consistent with a $t^{-0.5}$ decay over five months which is too shallow for a GRB afterglow (see Sect. 5.3) or a typical tidal disruption event which fades with $t^{-5 / 3}$ in the X-ray regime (Komossa 2015). The latter detection rules out the possibility that X6 is a GRB.

In archival PTF images we find two bright stars, hereafter referred to as S1 and S2, located close to the $90 \%$ error circle of X6. To look for fainter optical sources we obtained a Keck image in which a third object, O3, is detected (see Fig. A.2). The properties of the three potential optical counterparts are specified in Table A.1.

To search for short-lived optical emission, we analyze simultaneous UVOT observations. During the first XRT observation the UVOT observed in the $U$ band (Target ID 34357). We use the HEASOFT tool uvotdetect to measure the aperture flux within a circle with a radius of $3^{\prime \prime}$ centered around the best 
Table 3. XRT sources.

\begin{tabular}{lllllll}
\hline \hline Name & RA & Dec & $\begin{array}{l}\text { Exposure time } \\
(\mathrm{s})\end{array}$ & $\begin{array}{l}\text { Rate } \\
\text { (counts/s) }\end{array}$ & Alt. name & Object type \\
\hline X1 & 25.4909 & +39.3921 & 308 & $0.097 \pm 0.020$ & B2 0138+39B & Seyfert 1 Galaxy \\
X2 & 25.6546 & +40.3788 & 285 & $0.047 \pm 0.015$ & HD 10438 & Star \\
X3 & 25.5324 & +39.4129 & 324 & $0.035 \pm 0.012$ & V* OQ And & Variable Star \\
X4 & 26.7475 & +39.2575 & 284 & $0.024 \pm 0.011$ & 1RXS J-14658.4+391526 & Star \\
X5 & 25.0723 & +39.5886 & 221 & $0.029 \pm 0.014$ & HD 10169 & Star \\
X6 & 25.0107 & +39.6033 & 506 & $0.017 \pm 0.007$ & - & unknown \\
\hline
\end{tabular}

Notes. Coordinates are provided in J2000.

fit location of X6. This small radius was chosen to avoid contamination from the star S2. No source is detected and the $3 \sigma$ limit is $17.39 \operatorname{mag}_{\mathrm{AB}}$ which corresponds to a flux upper limit of $10^{-15} \mathrm{erg} \mathrm{s}^{-1} \mathrm{~cm}^{-2} \AA^{-1}$ at a wavelength of $3501 \AA$.

Considering all available observations, we identify two possible scenarios: X6 could either be an extreme stellar flare or it could be an obscured and distant AGN. We discuss the nature of X6 in more detail in Appendix A, where we come to the conclusion that it is not likely associated with the neutrino triplet.

Except for X-ray source X6, the Swift follow-up observations identified no unknown X-ray sources within the 50\%containment region of the neutrino triplet. Our upper limits on any source over this region are derived from the $0.3-1.0 \mathrm{keV}, 1-$ $2 \mathrm{keV}, 2-10 \mathrm{keV}$, and $0.3-10 \mathrm{keV}$ (full band) background maps. Background count rates for each bandpass were estimated from three regions, sampling the on-axis, off-axis, and field-overlap portions of the total exposure pattern; these provide a $3 \sigma$ countrate upper limit following the Bayesian method of Kraft et al. (1991). The upper limits were then multiplied by a factor of 1.08 to correct for the finite size of the aperture (a 20 pixel radius). The rate upper limits are converted to fluxes for each of two spectral models: a typical AGN spectrum in the X-ray band (a power law with photon index $\Gamma=-1.7, N_{\mathrm{H}}=3 \times 10^{20} \mathrm{~cm}^{-2}$ ) and a GRB spectrum (a power law with $\Gamma=-2, N_{\mathrm{H}}=3 \times 10^{21} \mathrm{~cm}^{-2}$ ). The range of resulting upper limits is listed in Table B.3. In Sect. 5.3 we compare the limits to detected GRB afterglows.

\subsection{Gamma-ray observations}

The position of the triplet was observed by the Fermi LAT about 30 min after the neutrino detection (see Sect. 4.3.1). Bad weather conditions in La Palma did not allow immediate observations with either MAGIC (Aleksić et al. 2016) or FACT (Anderhub et al. 2013) and the position is not observable for HESS. VERITAS observed the direction with a delay of one week (see Sect. 4.3.2) and the position was within HAWC's FoV at the arrival time of the triplet (see Sect. 4.3.3).

\subsubsection{Fermi Large Area Telescope}

The Fermi Gamma-ray Space Telescope consists of two primary instruments, the Large Area Telescope (LAT) and the GammaRay Burst monitor (GBM). The LAT is a pair-conversion telescope comprising a $4 \times 4$ array of silicon strip trackers and cesium iodide (CsI) calorimeters. The LAT covers the energy range from $20 \mathrm{MeV}$ to more than $300 \mathrm{GeV}$ with a FoV of $\sim 2.4$ steradian, observing the entire sky every two orbits $(\sim 3 \mathrm{~h})$ while in normal survey mode (Atwood et al. 2009). The GBM is comprised of 12 sodium iodide (NaI) and two bismuth germanate (BGO) scintillation detectors that have an instantaneous view of $70 \%$ of the sky. The NaI and BGO detectors are sensitive to emission between $8 \mathrm{keV}$ and $1 \mathrm{MeV}$, and $150 \mathrm{keV}$ and $40 \mathrm{MeV}$, respectively (Meegan et al. 2009).

The triplet location was occulted by the Earth at the detection time of the first neutrino event (T0). As a result, the GBM and LAT can place no constraints on the existence of a prompt gamma-ray transient coincident with the detection of the neutrino events. Within the period of $24 \mathrm{~h}$ before and after T0, there were a total of four reported GBM detections ${ }^{8}$. They were all separated by more than $50^{\circ}$ from the triplet location and an association can be excluded.

The region of interest entered the LAT field-of-view after roughly $1600 \mathrm{~s}$ and in the following we analyze the LAT data recorded within the days before and after the detection of the neutrino alert. We focussed on limiting the intermediate (hours to days) to long (weeks) timescale emission from a new transient source or flaring activity from a known gamma-ray emitter in the LAT energy range. We employed two different techniques to search for such emission in the LAT data; the Fermi All-sky Variability analysis (FAVA; Ackermann et al. 2013a) and a standard unbinned likelihood analysis. FAVA is an all-sky photometric analysis in which a region of the sky is searched for deviations from the expected flux based on the mission-averaged data. The unbinned likelihood analysis is the standard method of detecting and characterizing sources in the LAT data and is described in more detail in Abdo et al. (2009). We additionally employed a profile likelihood method described in Ackermann et al. (2012) to calculate upper limits in situations when no significant excess emission is detected.

The FAVA search was performed on $24 \mathrm{~h}$ timescales bracketing T0, covering the periods of [T0-24h to T0], [T0 $-12 \mathrm{~h}$ to $\mathrm{T} 0+12 \mathrm{~h}$ ], and [T0 to $\mathrm{T} 0+24 \mathrm{~h}$ ] (see Table 4 ). A single week-long timescale was also searched, covering the period of [T0-2.15 days to T0+4.85 days]. The FAVA analysis selects flares that have a significance of $6 \sigma$ above the mission average emission at the location. Within the analyzed time windows no such flare was detected at the triplet location.

An examination of the second FAVA catalog (2FAV, paper in preparation), which lists all flaring sources detected in the LAT data on weekly timescales over the course of the entire mission, shows only one period of flaring activity within the $90 \%$ error circle of the triplet location ${ }^{9}$. This period of activity was between 2009-08-31 and 2009-09-07 and was associated with 3FGL J0156.3+3913 which is a blazar candidate of uncertain type (Acero et al. 2015). No further activity from this source has been detected by FAVA.

\footnotetext{
8 http://gcn.gsfc.nasa.gov/fermi_grbs.html

9 http://fermi.gsfc.nasa.gov/ssc/data/access/lat/FAVA/ LightCurve.php?ra=26.1\&dec=39.5
} 


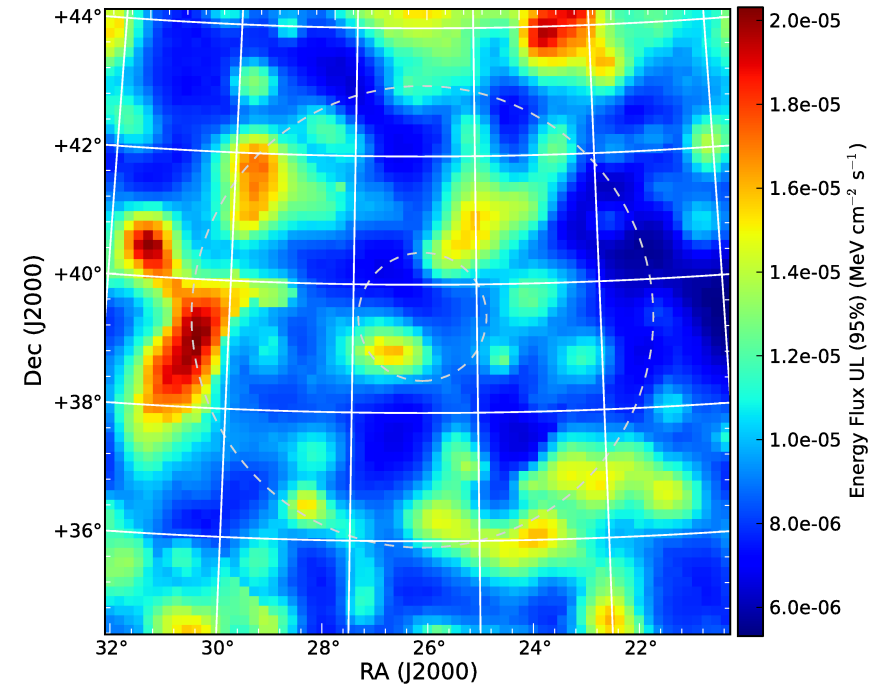

(a) The Fermi LAT likelihood ratio test statistic (TS) within the region of interest. The significance of fluctuations above the expected background scales roughly with $\sqrt{\mathrm{TS}}$.

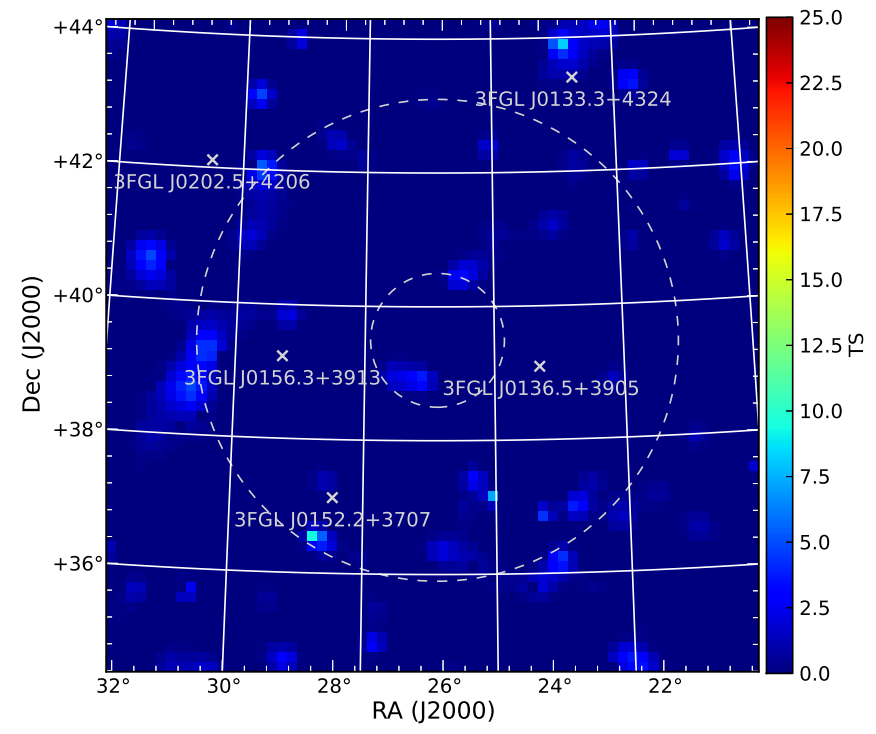

(b) Fermi LAT 95\% upper limits on the flux in the $100 \mathrm{MeV}$ to $100 \mathrm{GeV}$ energy range. Crosses indicate the locations of known Fermi sources.

Fig. 4. Fermi LAT results from the unbinned likelihood analysis within the region of interest using all data within 14 days of neutrino detection. The dashed circles show the $50 \%$ and $90 \%$ error circles of the neutrino triplet.

Table 4. Fermi LAT flux upper limits.

\begin{tabular}{llllll}
\hline \hline Interval & Duration & $\begin{array}{l}\text { Start date } \\
\text { (UTC) }\end{array}$ & $\begin{array}{l}\text { End date } \\
\text { (UTC) }\end{array}$ & $\begin{array}{l}\text { Median U.L. (95) } \\
\left(\mathrm{ph} \mathrm{cm}^{-2} \mathrm{~s}^{-1}\right)\end{array}$ & $\begin{array}{l}\text { Median U.L. (95) } \\
\left(\mathrm{GeV} \mathrm{cm}^{-2} \mathrm{~s}^{-1}\right)\end{array}$ \\
\hline$T_{\text {FAVA1 }}$ & $24 \mathrm{~h}$ & $2016-02-1719: 21: 32$ & $2016-02-1819: 21: 32$ & - & - \\
$T_{\text {FAVA2 }}$ & $24 \mathrm{~h}$ & $2016-02-1619: 21: 32$ & $2016-02-1719: 21: 32$ & - & - \\
$T_{\text {FAVA3 }}$ & $24 \mathrm{~h}$ & $2016-02-1707: 21: 32$ & $2016-02-1807: 21: 32$ & - & - \\
$T_{\text {FAVA4 }}$ & 7 days & $2016-02-1515: 43: 35$ & $2016-02-2215: 43: 35$ & - & - \\
\hline$T_{\text {Like1 }}$ & $6 \mathrm{~h}$ & $2016-02-1719: 21: 32$ & $2016-02-1801: 21: 32$ & $3.32 \times 10^{-7}$ & $1.82 \times 10^{-7}$ \\
$T_{\text {Like2 }}$ & $12 \mathrm{~h}$ & $2016-02-1719: 21: 32$ & $2016-02-1807: 21: 32$ & $1.86 \times 10^{-7}$ & $1.01 \times 10^{-7}$ \\
$T_{\text {Like3 }}$ & $24 \mathrm{~h}$ & $2016-02-1719: 21: 32$ & $2016-02-1819: 21: 32$ & $1.27 \times 10^{-7}$ & $6.96 \times 10^{-8}$ \\
$T_{\text {Like4 }}$ & $24 \mathrm{~h}$ & $2016-02-1619: 21: 32$ & $2016-02-1719: 21: 32$ & $1.15 \times 10^{-7}$ & $6.30 \times 10^{-8}$ \\
$T_{\text {Like5 }}$ & $24 \mathrm{~h}$ & $2016-02-1707: 21: 32$ & $2016-02-1807: 21: 32$ & $1.11 \times 10^{-7}$ & $6.08 \times 10^{-8}$ \\
$T_{\text {Like6 }}$ & 14 days & $2016-02-1719: 21: 32$ & $2016-03-0219: 21: 32$ & $1.73 \times 10^{-8}$ & $9.48 \times 10^{-9}$ \\
\hline
\end{tabular}

Notes. A summary of the FAVA and likelihood analysis timescales. FAVA does not provide flux upper limit estimates. The upper limit estimates quoted for the likelihood analysis are the median 95\% C.L. considering all upper limits within the 90\% error circle. They have been obtained for the energy range from $100 \mathrm{MeV}$ to $100 \mathrm{GeV}$ and a spectral index of $\Gamma=-2.1$ has been assumed.

The unbinned likelihood analysis was performed using the standard LAT analysis tools (ScienceTools version v10r01p0) $)^{10}$ by modeling all photons within a region of interest (ROI) with a radius of $12^{\circ}$, covering an energy range of $100 \mathrm{MeV}$ to $100 \mathrm{GeV}$, and utilizing the P8R2_TRANSIENTR020_V6 event class and the corresponding instrument response functions. For the purposes of this analysis, all modeled sources were fixed to their cata$\log$ values, while the normalization of the Galactic and diffuse isotropic components of the fit were allowed to vary. Because of the uncertainty in the triplet location, this analysis was repeated over a $10^{\circ} \times 10^{\circ}$ grid of coordinates with $0.15^{\circ}$ binning.

This search was performed over a variety of timescales, ranging from $6 \mathrm{~h}$ to 14 days (Table 4 ). The resulting significance maps show no emission in excess of the expected background on any of the timescales considered. For each bin in the coordinate

\footnotetext{
10 http://fermi.gsfc.nasa.gov/ssc/
}

grid, we calculated the $95 \%$ confidence levels (C.L.) upper limit on the photon flux of a candidate point source with a fixed spectral index of $\Gamma=-2.1$. This value is appropriate for both AGN (compare Ackermann et al. 2015) and GRBs (Ackermann et al. 2013b; Gruber et al. 2014) and is used as the standard value when searching for GRBs. An example of the significance and energy upper limit maps for the $\mathrm{T} 0+14$ day timescale is shown in Fig. 4. The median photon flux and energy flux upper limits calculated for each timescale are listed in Table 4.

\subsubsection{Very Energetic Radiation Imaging Telescope Array System}

The Very Energetic Radiation Imaging Telescope Array System (VERITAS) is a ground-based instrument for VHE gamma-ray astronomy with maximum sensitivity in the $80 \mathrm{GeV}$ to $30 \mathrm{TeV}$ 
range. It is located at the Fred Lawrence Whipple observatory in southern Arizona $\left(31^{\circ} 40^{\prime} \mathrm{N}, 110^{\circ} 57^{\prime} \mathrm{W}\right)$ at an altitude of $1.3 \mathrm{~km}$ above sea level. The array consists of four 12-m-diameter imaging air Cherenkov telescopes each equipped with a camera containing 499 photomultiplier tubes (PMTs) covering a $3.5^{\circ}$ FoV. Full details of the VERITAS instrument performance and sensitivity are given in Park (2015).

At the time the triplet detection was communicated to VERITAS, the Moon was approaching its full phase and the night sky was too bright to safely operate the sensitive PMT cameras. It is, however, not uncommon for some variable VHE sources such as AGN to exhibit extended periods of intense flaring activity that can be detected days after the source has reached its peak flux (Dermer \& Giebels 2016). Observations were started eight days after the detection of the neutrino events on 2016-02-25, when VERITAS observed the triplet location between 02:32 and 03:20 UTC. Additional observations were taken on 2016-02-26 between 02:36 and 03:43 UTC. The combined exposure time during these two nights was $62.8 \mathrm{~min}$, after quality cuts were applied. These observations were carried out in the normal "wobble" mode, where the pointing direction of the telescopes is offset from the source position to allow for simultaneous measurement of the background (Berge et al. 2007). A wobble offset of $0.7^{\circ}$ was selected to cover a larger region of sky given the uncertainty in the averaged triplet position.

An analysis of the VERITAS data showed no significant gamma-ray excess in the triplet region of interest (see Fig. 5). Consequently, differential flux upper limits were calculated at the $95 \%$ confidence level in four energy bins for a gamma-ray point source located at the averaged triplet position and are given in Table B.4. Furthermore, no new gamma-ray sources were detected anywhere within the triplet $50 \%$ error region or within the VERITAS FoV.

The only known VHE source in the vicinity of the triplet is the high-synchrotron-peaked blazar RGB J0136+391 ${ }^{11}$ (also 3FGL J0136.5+3905; see Fig. 4a). It has an approximate angular distance of $1.6^{\circ}$ from the triplet central position and was not detected during the VERITAS observations (see Sect. 5.4 for further discussion of this source). Therefore, the data show no indication of a persistent VHE gamma-ray source, or a high state of RGB J0136+391, which could be associated with the neutrino events.

\subsubsection{The High Altitude Water Cherenkov observatory}

The High Altitude Water Cherenkov (HAWC) observatory is an array of 300 detectors, each filled with approximately 200000 liters of purified water and instrumented with four photo-multiplier tubes. Light-tight bladders provide optical isolation. The observatory is optimized to detect Cherenkov light from extensive air showers produced by gamma-ray primaries at energies between $100 \mathrm{GeV}$ and $100 \mathrm{TeV}$. HAWC is located in the state of Puebla, Mexico at an altitude of $4100 \mathrm{~m}\left(97.3^{\circ} \mathrm{W}\right.$, $\left.19.0^{\circ} \mathrm{N}\right)$. HAWC operates continuously and has an average down time due to maintenance of only $\sim 5 \%$. A wide FoV, approximately defined by a cone with an opening angle of $45^{\circ}$ from zenith, spans the declination range of $-26^{\circ}$ to $+64^{\circ}$ and rotates with the Earth through the full range of right ascension every day. For a detailed description of the array and analysis methods see Abeysekara et al. (2017b).

At the detection time of the neutrino triplet, its position had just entered HAWC's FoV. HAWC was operating normally and

11 http: //tevcat. uchicago . edu/?mode $=1$; id=244

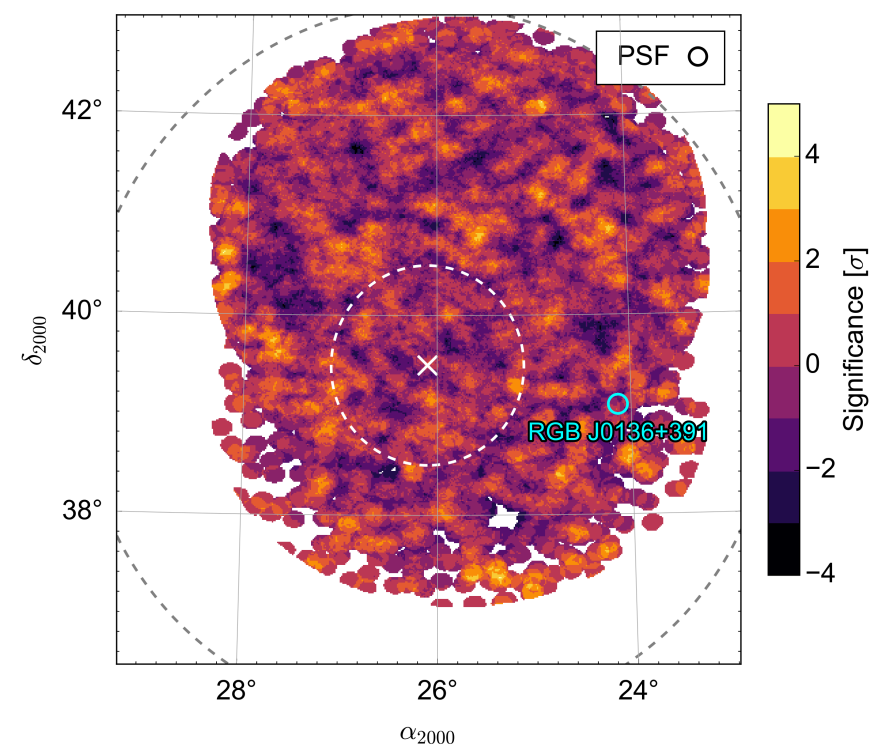

Fig. 5. Significance sky map for the VERITAS observations of the neutrino triplet region. The dashed white (gray) line indicates the 50\% $(90 \%)$ error circle for the triplet. No gamma-ray excess was detected in the FoV. The known VHE source RGB J0136+391 (also known as 3FGL J0136.5+3905; compare Fig. 4a) is located approximately $1.6^{\circ}$ away from the triplet central position.

observed the full transit $\left(\sim 6 \mathrm{~h}\right.$ at zenith angles $\left.<45^{\circ}\right)$ of the triplet location between 19:15 UTC on 2016-02-17 and 01:30 UTC on 2016-02-18. HAWC data are being continuously reconstructed on computers at the array site with an average time lag of approximately $4 \mathrm{~s}$ and were immediately available for a follow-up analysis when the IceCube alert was received.

A scan of the region around the triplet coordinates was performed with the standard HAWC maximum-likelihood technique, using nine energy-proxy analysis bins that sort data according to the air shower size (Abeysekara et al. 2016). The analysis bins account for the varying angular resolution and background suppression efficiency. For each bin, the event count in each pixel of a HEALPix (Górski et al. 2005) map is compared to a prediction composed of the average, smoothed background of cosmic rays measured from data and the simulated expectation of gamma-ray events from a point-like source. The signal expectation includes the modeling of the angular resolution, which improves with energy from $\sim 1^{\circ}$ to $<0.2^{\circ}$ in the range from 1 to $100 \mathrm{TeV}$. The differential flux in each analysis bin is described by a power law with a photon index of $\Gamma=-2.7$, which is the standard value used for HAWC point-source searches. This index also corresponds to the average of detected TeVCat sources (Abeysekara et al. 2017a). Leaving only the normalization $N_{0}$ as a free parameter, a likelihood maximization over all bins and pixels was performed for all locations in a $9^{\circ} \times 9^{\circ}$ area with a grid spacing of $0.06^{\circ}$. This scan revealed no significant excess with a pre-trial significance above $5 \sigma$ and the results are fully compatible with a pure background hypothesis. The resulting sky map is presented in Fig. 6, showing significance in standard deviations calculated as $\sqrt{\mathrm{TS}}$, where TS is the standard test statistic from the likelihood ratio test.

Given the lack of a source candidate, we derived gammaray flux limits for the combined average neutrino direction, $\mathrm{RA}=26.1^{\circ}$, Dec $=39.5^{\circ}$. The resulting limits are listed in Table B.5 and shown in Sect. 5. These upper limits were calculated separately for five intervals of width 0.5 in $\log (E / \mathrm{TeV})$ 


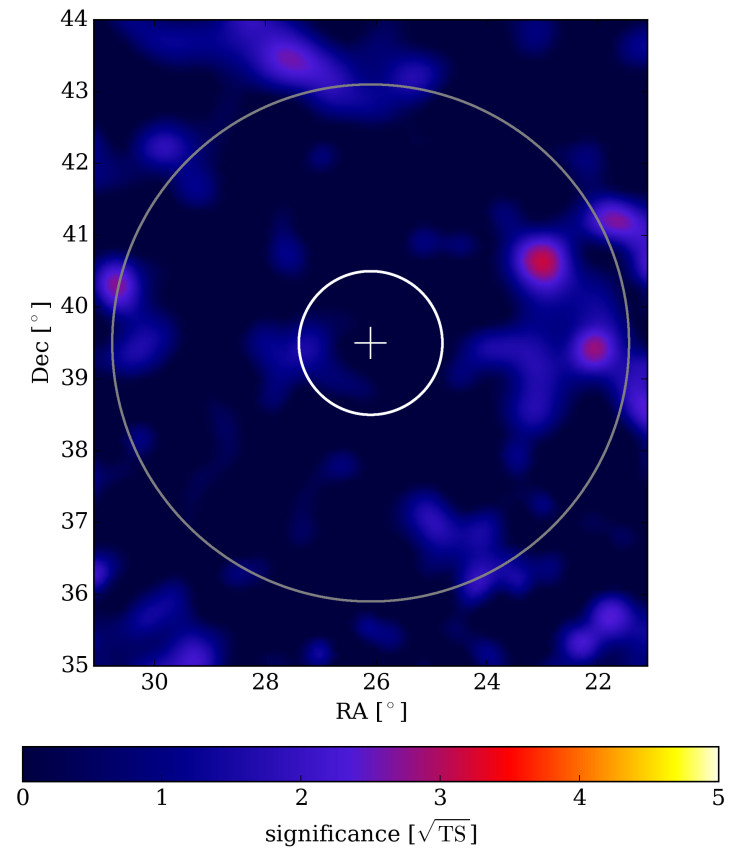

Fig. 6. HAWC $500 \mathrm{GeV}$ to $160 \mathrm{TeV}$ significance sky map for data collected over one transit between 19:18 UTC on 2016-02-17 and 01:31 UTC on 2016-02-18, centered at RA $=26.1^{\circ}, \mathrm{Dec}=39.5^{\circ}$. The IceCube $50 \%$ (white) and $90 \%$ (gray) error circles are also shown.

by modeling a flux that is non-zero only within each interval and using a scan of the likelihood space to determine the one-sided 95\% C.L. value. The limits correspond to the normalization $N_{0}$ of a power law with a photon index of $\Gamma=-2$. We checked that the normalization in the center of each interval did not change when varying $\Gamma$ between 0 and -3 and conclude that the limits are independent of any spectral assumption. The energy range covered by these limits extends from $500 \mathrm{GeV}$ to $160 \mathrm{TeV}$. A discussion of systematic uncertainties of HAWC flux measurements can be found in Abeysekara et al. (2017b). These systematic uncertainties are not incorporated into the limits.

For better comparison to other, non-coincident observations in this paper, we also analyzed the 14 day period starting with the transit during the alert and ending on 2016-03-01, 00:30 UTC. Detector down time and quality cuts led to the exclusion of three transits (February 22, 25, and 26) due to marginal coverage. No significant excess was found in the combined data for the eleven full transits of the multiplet location and we also calculated limits for this period.

Since HAWC had been operating for more than a year before the alert and continues to provide daily monitoring, we also analyzed the integrated data from 508.2 transits of the triplet location between 2014-11-26 and 2016-06-02. No significant excess was found within the IceCube $90 \%$ error radius and we derived a quasi-differential limit for the average flux at the central location during this period, included in Table B.5.

\section{Discussion}

We now draw conclusions from the non-detections during the follow-up observations and discuss the sensitivity of our program to a potential astrophysical multiplet source. An overview of the obtained limits is shown in Fig. 7.

As shown in Sect. 3.2, the detection of a neutrino triplet is expected once every $\sim 13.7 \mathrm{yr}$ from random coincidences of atmospheric background events and we cannot exclude such a chance alignment as the source of the triplet. However, the neutrino multiplet could also stem from a transient neutrino source which emitted a $\sim 100 \mathrm{~s}$ burst of $\mathrm{TeV}$ neutrinos. Since three neutrinos are detected, a potential source has to be either close-by or extremely energetic. Possible transient source classes include CCSNe with an internal jet, GRBs or AGN flares.

\subsection{Distance of an astrophysical neutrino source}

We used a simulated population of transient neutrino sources to estimate their typical distances, which is important for the interpretation of the follow-up observations. The astrophysical neutrino flux, detected at $\mathrm{TeV} / \mathrm{PeV}$ energies, is best described by an $E^{-2.5}$ spectrum (Aartsen et al. 2015a) ${ }^{12}$. We adopt this spectral shape as well as the measured normalization and consider simulated neutrino events which passed the event selection of the follow-up program. We expect the detection of 600 astrophysical muon neutrinos per year from the Northern sky. For this calculation, we extrapolated the measured neutrino spectrum down to $10 \mathrm{GeV}$, below the IceCube sensitivity threshold. If we were only to consider events above $10 \mathrm{TeV}$ where the astrophysical flux has been measured (Aartsen et al. 2015a), we would expect the detection of 200 events per year. The large number of expected astrophysical neutrino events results from the broad, inclusive event selection of the follow-up program which aims to include all well-reconstructed track events.

We simulate a population of transient neutrino sources that accounts for the complete astrophysical neutrino flux. The cosmic star-formation rate approximately describes the redshift distributions of several potential neutrino sources, like CCSNe (Cappellaro et al. 2015) and GRBs (Wanderman \& Piran 2010; Salvaterra et al. 2012; Krühler et al. 2015) which however tend to be located at slightly larger redshifts. We simulated a source population using the star-formation rate of Madau \& Dickinson (2014) and calculated for each source the probability of detecting it with a certain number of neutrinos after applying the event selection of the follow-up program. We find that a source detected with a single neutrino is located at a median redshift of $z=1.1$, as shown in Fig. 8 .

To calculate the distance to a source detected with multiple neutrinos, we have to simulate how bright the individual sources are. We assume a population with a local source rate of $10^{-6} \mathrm{Mpc}^{-3} \mathrm{yr}^{-1}$, which corresponds to $\sim 1 \%$ of the CCSN rate (see e.g., Strolger et al. 2015). If this population accounts for the astrophysical neutrino flux, we expect the detection of one neutrino triplet (or higher multiplet) per year. The rate of multiplet alerts, however, strongly depends on the spectral shape and considered energy range of the neutrino flux. We further assumed that the luminosity fluctuations between the neutrino sources follow a log-normal distribution with a width of one astronomical magnitude, which is comparable to the luminosity spread of CCSNe in optical light at optical wavelengths.

Figure 8 shows that the source of a neutrino doublet has a median redshift of $z=0.06$ and the median redshift of a triplet source is $z=0.023$. We note that these results strongly depend

12 We note that a significantly shallower power law index of $E^{-2.13}$ was measured at energies above $\sim 100 \mathrm{TeV}$ by Aartsen et al. (2016a). The astrophysical neutrino spectra detected in both analyses are however consistent at those high energies. Like Aartsen et al. (2016a) we therefore interpret this apparent discrepancy as an indication of a break in the neutrino spectrum. The steep spectral index of $E^{-2.5}$ measured in (Aartsen et al. 2015a) is more relevant for this work because it extends to lower energies, down to $\sim 10 \mathrm{TeV}$. 


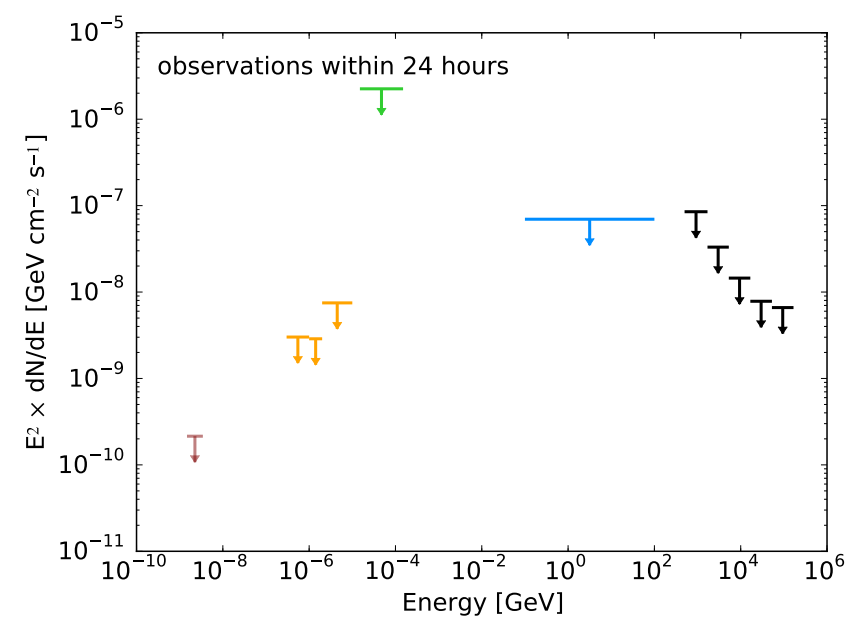

(a) Limits on short transients.

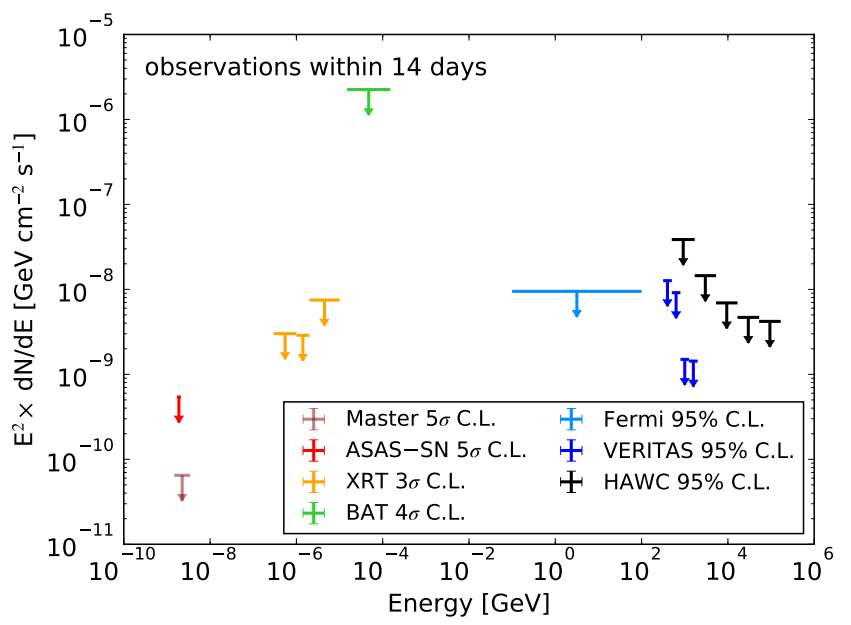

(b) Limits on longer lasting transients.

Fig. 7. Flux upper limits from the multiwavelength observations. The confidence level varies between the different observations as indicated in the legend and some limits depend on the assumed source spectrum (Swift XRT and BAT $\Gamma=-2$ and Fermi LAT $\Gamma=-2.1$; see Sect. 4). For the optical telescopes, the limit corresponding to the deepest observation is shown, while for the other instruments, all analyzed data were combined. The limit for the Swift BAT is purely based on the observation taken $100 \mathrm{~s}$ after the detection of the first neutrino (compare Sect. 4.2.1) and hence applies to prompt gamma-ray emission. Follow-up observations were triggered $22 \mathrm{~h}$ after the detection of the neutrino triplet.

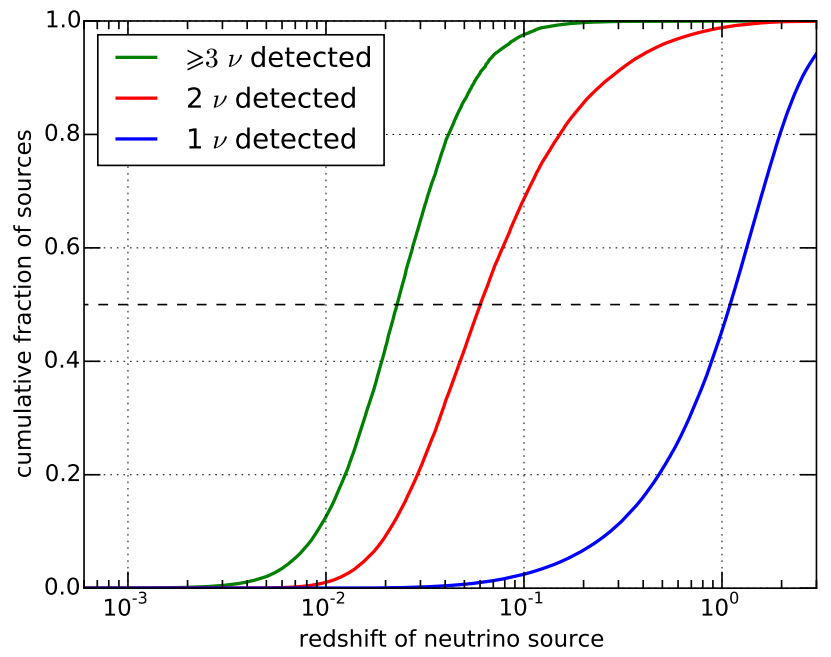

Fig. 8. Probability of detecting a neutrino source within a certain redshift. The figure was generated by simulating a population of transient neutrino sources with a density of $10^{-6} \mathrm{Mpc}^{-3} \mathrm{yr}^{-1}$ distributed in redshift according to the star-formation rate and normalized to produce the detected astrophysical neutrino flux. Sources detected with only one single neutrino are on average far away (median redshift of 1.1), while sources detected with three or more neutrinos must be located nearby.

on the spectral shape of the astrophysical neutrino flux. Considering only neutrino events with an energy above $10 \mathrm{TeV}$, the source rate that yields one triplet per year is $3 \times 10^{-8} \mathrm{Mpc}^{-3} \mathrm{yr}^{-1}$ and the median redshift of a triplet source increases to $z=0.07$. If we would adopt the spectral index of $E^{-2.13}$ (Aartsen et al. 2016a), the source rate would be $2 \times 10^{-9} \mathrm{Mpc}^{-3} \mathrm{yr}^{-1}$ which would result into a median redshift of $z=0.17$ for a triplet source.

Similar calculations apply to a population of GRBs, AGN, or blazars, which, however, have different source densities, redshift distributions, and luminosity functions. We also note that the duration of $100 \mathrm{~s}$ to which our search is sensitive, does not enter these estimates and the distance calculation applies equally to steady sources.

In summary, we estimate that a CCSN detected with three neutrinos has a median redshift of $z=0.023$ or less assuming that $\mathrm{CCSNe}$ account for the complete astrophysical neutrino flux. Typical CCSNe below this redshift are easily detected with optical telescopes if they are not unusually faint or strongly affected by absorption. Even without extrapolating the astrophysical neutrino spectrum to lower energies or when adopting the hard spectral shape measured at high energies the SN would likely still be detectable (compare Sect. 5.2).

\subsection{Supernovae}

Figure 9 shows the constraints derived from the optical observations before and after the alert. As a comparison we plot the light curve of the bright Type Ic broadlined supernova SN 1998bw which accompanied GRB 980425 (Galama et al. 1998). A similar supernova would be detectable out to a redshift of $\sim 0.15$ which is much further than the expected redshift of a triplet source (compare Fig. 8).

In follow-up observations of the most significant neutrino doublet detected so far, a fading Type IIn supernova was found (Aartsen et al. 2015b). A comparable event can be ruled out with the optical observations shown in Fig. 9. We hence can exclude a nearby supernova unless it was unusually dim or heavily obscured.

\subsection{Gamma-ray bursts}

For CCSNe, we assumed that the source of a triplet must be close-by, following calculations in Sect. 5.1. GRBs are much less frequent than CCSNe which means that they are on average located at larger distances. Another difference is that the luminosity differences between individual GRBs can be extreme in gamma-rays (see e.g., Wanderman \& Piran 2010) which makes it likely that the neutrino luminosities also differ widely. Both effects boost the probability of finding a burst that is brighter (in neutrinos) than any burst that happened since the start of the follow-up program. We therefore do not restrict our search to very close-by GRBs.

To estimate whether or not a GRB would be detectable in the follow-up observations, we compare the upper limits to Swift gamma-ray light curves and X-ray afterglows in Fig. 10a. The light curves in the $15-50 \mathrm{keV}$ energy band were obtained from 


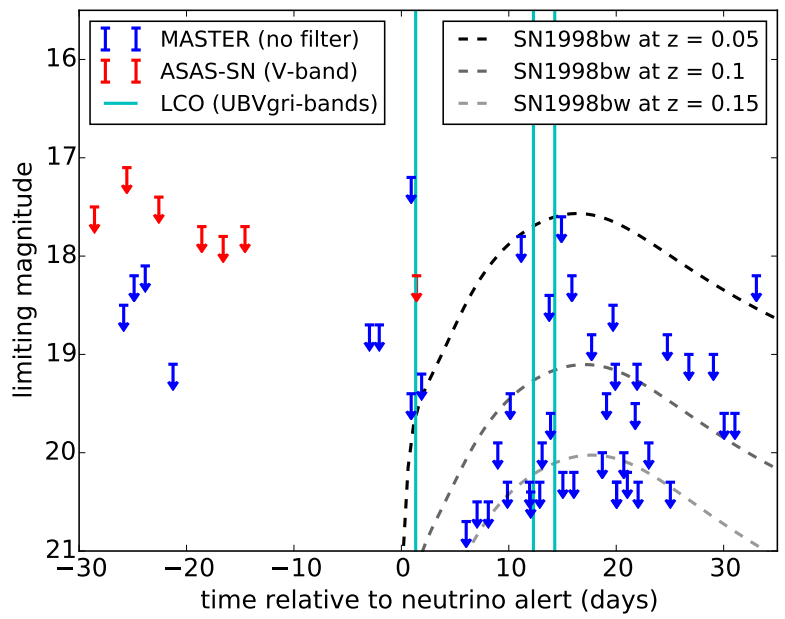

Fig. 9. Optical $5 \sigma$ limiting magnitudes from Table B.1 and described in Sect. 4.1. LCO epochs (from Table B.2) are shown as vertical lines. At these times, observations in the UBVgri bands were obtained, however no image subtraction was done. We overplot, as an example, the $V$-band light curve of SN 1998bw, which was associated with GRB 980425. The synthetic light curves of SN 1998bw have been created using the method presented in Cano (2014).

the UK Swift Science Data Centre ${ }^{13}$ (Evans et al. 2010). The median fluence deposited in this band is $41 \%$ of the total fluence for GRBs in the Swift GRB catalog ${ }^{14}$. We use this average factor to scale the fluxes to the full energy range of $15-150 \mathrm{keV}$ for which the BAT limit was calculated in Sect. 4.2.1. The central line corresponds to the median flux and the band contains $80 \%$ of all GRB. The light curves are not corrected for the redshift and nondetections have been removed. The distribution is hence heavily biased and provides only a rough estimate for typical GRB light curves.

The limits from the Swift BAT and XRT observations (see Sect. 4.2) are comparable to the fluxes of bright GRBs. A brighter-than-average GRB would have been detected, but most GRBs are fainter than the limits. Neutrino multiplet alerts are usually sent to the XRT without delay and the XRT observations typically start within half an hour of the neutrino signal being detected (Evans et al. 2015) when GRBs are on average more than two orders of magnitude brighter.

We checked the archival data of the InterPlanetary Network (IPN; Hurley et al. 2010) for a burst in temporal coincidence with the triplet. No confirmed ${ }^{15}$ or unconfirmed ${ }^{16}$ GRB was detected on the day of the triplet alert (Hurley, Priv. Comm.).

GRB afterglows are also detectable in optical observations. In Fig. 10b we compare our observations to a large sample of optical GRB afterglows (Kann et al. 2010, 2011, 2017). As before, the shaded band includes $80 \%$ of all GRBs in the sample. Only the brightest afterglows are detectable in the earliest optical observations. Nearby GRBs have been found to be accompanied by a Type Ic broadlined SN (Cano et al. 2017) and as shown in Sect. 5.2 a nearby SN is disfavored. GRBs with a slightly misaligned jet might in addition produce orphan afterglows which could be detectable in optical (see e.g., Zou et al. 2007;

\footnotetext{
13 http://www.swift.ac.uk/burst_analyser/

${ }^{14}$ http://heasarc.gsfc.nasa.gov/W3Browse/swift/ swiftgrb.html

15 http://heasarc.gsfc.nasa.gov/w3browse/all/ipngrb. html

16 http://www.ssl. berkeley. edu/ipn3/cosmic1.txt
}

Ghirlanda et al. 2015; Kathirgamaraju et al. 2016) or in X-ray observations (see e.g., Evans et al. 2016; Sun et al. 2017).

Correlation analyses of detected GRBs with IceCube neutrino events show that gamma-ray bright GRBs are not the main sources of the astrophysical neutrino flux (Abbasi et al. 2012a; Aartsen et al. 2015c, 2016c). These limits however only apply to gamma-ray bright sources which are routinely detected with current gamma-ray satellites. To gain sensitivity to low-luminosity GRBs, which might be missed in gamma rays, quick X-ray and optical observations are essential. In addition, early optical follow-up observations can be used to look for rapidly fading transients without associated gamma-ray emission (like the object found by Cenko et al. 2013) or for GRBs that were missed by gamma-ray detectors (Cenko et al. 2015).

In summary we conclude that a bright GRB likely would have been detected by both the BAT and the Swift XRT while a typical GRB is too faint. Moreover, there is a class of lowluminosity GRBs (Liang et al. 2007) which could be below the detection threshold of existing instruments even when occurring at low redshifts. The accompanying $\mathrm{SNe}$ of such objects might however be detectable (compare Sect. 5.2).

\subsection{Active galactic nuclei}

The durations of typical AGN flares observed in gamma rays range from minutes to several weeks. The time scale of $100 \mathrm{~s}$ is hence short and implies that the neutrinos have to be emitted from a very small region of the jet even when taking into account relativistic beaming. The dedicated gamma-ray follow-up program of IceCube searches for neutrino emission on time scales of up to three weeks (Kintscher 2016; Aartsen et al. 2016b). Currently the gamma-ray follow-up program searches for emission from sources on a predefined source list and none of those sources is consistent with the triplet direction.

The Swift XRT observations resulted in the detection of one known AGN (X1) and one AGN candidate (X6) within the 50\% error circle (see Sect. 4.2.2 and Appendix A). X1 is a blazar but does not exhibit flaring compared to X-ray observations taken in 2010 and 2011. X6 fades away following the neutrino alert, but is not very bright overall (see Appendix A) and remains undetected in gamma rays.

No flares were detected in gamma rays by the Fermi LAT, VERITAS, or HAWC. The three Fermi LAT sources within the $90 \%$ error circle of the event did not show a significant flux excess within the weeks before and after the alert. 3FGL J0156.3+3913 underwent flares in 2009, but was inactive at the time of the neutrino alert and 3FGL J0152.2+3707 has been classified as a blazar candidate of uncertain type (Acero et al. 2015).

The third source, RGB J0136+391 (or 3FGL J0136.5+3905), is a high frequency peaked $\mathrm{BL}$ Lac object at a redshift of $>0.4$ (inferred from the non-detection of its host galaxy by Nilsson et al. 2012). It was detected in VHE gamma rays by MAGIC in November 2009 with an observation time of $6.5 \mathrm{~h}^{17}$ (see also the non-detection by VERITAS at a similar time; Aliu et al. 2012). During the VERITAS observation eight days after the neutrino alert the source was not detected with $\sim 1 \mathrm{~h}$ of observation time (see Sect. 4.3.2). The source hence did not undergo a very bright and long-lasting flare. A shorter or less luminous flare is not excluded, even though no variability was detected by the Fermi LAT during this period (see Sect. 4.3.1).

\footnotetext{
${ }^{17}$ https://www.mpi-hd.mpg.de/hd2012/pages/ presentations/Mazin.pdf
} 


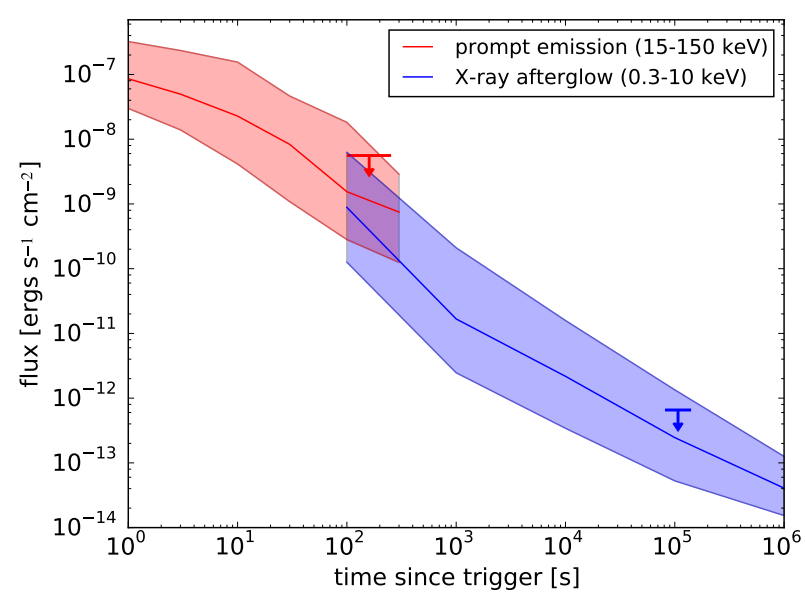

(a) Gamma-ray and X-ray GRB light curves.

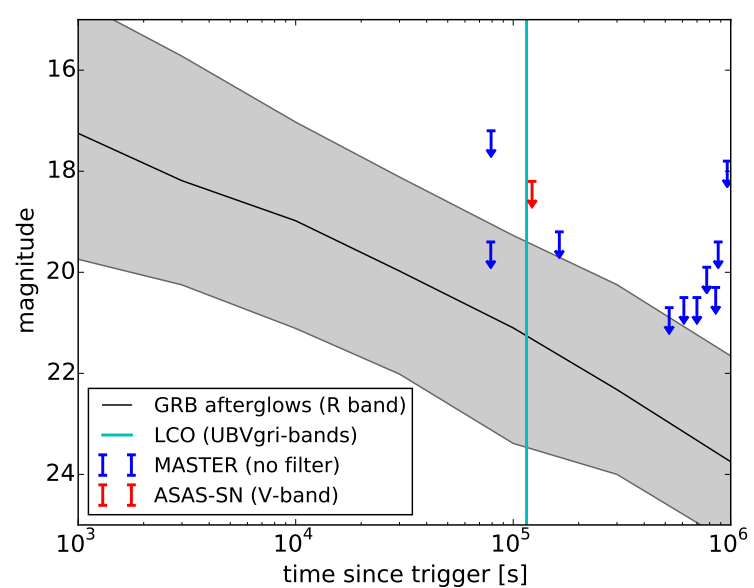

(b) Optical GRB light curves.

Fig. 10. The shaded bands show the gamma-ray and X-ray light curves of detected GRBs (left) and optical afterglow light curves (right). The central line shows the median flux at the indicated time and the shaded bands include $80 \%$ of all GRBs (i.e., the $10 \%$ brightest and faintest afterglows are above or below the band, respectively). The arrows show the flux upper limits from the X-ray and optical follow-up observations (see Sects. 4.1 and 4.2 for details).

To estimate how likely it is to find an unrelated VHE source within the $90 \%$ error circle of this neutrino alert we consider all AGN in the Northern sky that are detected in VHE gamma rays. The 60 sources in the $\mathrm{TeVCat}^{18}$ yield a probability of $\sim 6 \%$ of finding a source within $3.6^{\circ}$ of a random position. This rough estimate does not consider that neither the neutrino alerts nor the detected VHE sources are distributed randomly over the sky. It indicates, however, that the presence of RGB J0136+391 could be a coincidence.

We conclude that there is no evidence for AGN flares within the region of interest. We derived flux upper limits for two time ranges using observations taken within a period of $24 \mathrm{~h}$ and 14 days after the neutrino detection. The limits in the different wavelength regimes are shown in Fig. 7. It is unclear whether or not an AGN flare below the derived limits can yield a large neutrino flux.

\section{Summary}

For the first time, the IceCube follow-up program was triggered by three neutrinos within $100 \mathrm{~s}$ and with reconstructed directions consistent with a point source origin. Such an alert is expected from the coincidence of background events once every $13.7 \mathrm{yr}$. Considering that the program has been running since December 2008 in different configurations, the probability of detecting one or several triplets from atmospheric background is $32 \%$. When an alternative event reconstruction algorithm (Spline MPE) is applied, the event directions have larger angular separations and the multiplet would not have been considered interesting. This is an additional indication that the multiplet probably is not astrophysical.

Even so, the triplet is the most significant neutrino multiplet detected since the beginning of the follow-up program and follow-up observations were obtained in different wavelength regimes to search for a potential electromagnetic counterpart (see Sect. 4). No transient source was detected in the optical or gamma-rays regimes. The Swift XRT detected one highly variable X-ray source whose nature remains unknown (see Appendix A for a detailed discussion). As described in Sect. 4.2.2 this source is not consistent with a GRB. It could be a flaring AGN which however would not be very bright and is not

\footnotetext{
18 Status of 2017-01-04.
}

detected in gamma rays. We therefore conclude that this X-ray source is most likely not connected to the neutrinos.

Our optical observations are sufficient to rule out a nearby CCSN (see Sect. 5.2). A bright GRB would likely have been detected both in the Swift XRT observations and by the Swift BAT which serendipitously observed the location within minutes of the alert (see Sect. 5.3). However, low-luminosity GRBs might be too dim to be detectable even if they are located at low redshifts. No flaring AGN were found in either X-rays, gamma rays, or very-high-energy gamma rays. We conclude that no likely counterpart was identified in follow-up observations. Since the neutrino alert is consistent with background (see Sect. 3.2) we cannot place new constraints on astrophysical models for neutrino emission.

This work demonstrates that the IceCube follow-up program is able to trigger observations in near real-time to search for transient neutrino sources. While this alert was not triggered automatically, causing a delay of $22 \mathrm{~h}$, the system typically issues alerts within $\sim 1 \mathrm{~min}$, such that even rapidly fading transients are observable. Using additional serendipitous observations we demonstrate in Sect. 5 that the program is well suited to testing several suggested source classes.

We are planning to replace the fixed cuts used currently in the optical follow-up program (compare Sect. 2.3) with a likelihood search. This will increase the sensitivity and allow us to search for sources that last longer than $100 \mathrm{~s}$. A global network of optical telescopes, including ASAS-SN, LCO, MASTER, and the upcoming Zwicky Transient Facility (Bellm 2014), will moreover result into much better data coverage compared to previous years.

In the case of an astrophysical multiplet detection, the follow-up network employed here and in its future extension should enable the detection of its electromagnetic counterpart and hence identification of a neutrino source. Moreover, some of the methods presented here are readily generalizable to searches for counterparts of high-energy single neutrino events or for follow-up observations of gravitational wave events.

Acknowledgements. Neil Gehrels sadly died during the late stage of the production of this paper. As Swift PI he was an enthusiastic supporter of multi-messenger observations; he will be sorely missed. The IceCube Collaboration acknowledges the support from the following agencies: US National Science Foundation-Office of Polar Programs, US National Science FoundationPhysics Division, University of Wisconsin Alumni Research Foundation, the 
IceCube et al.: Follow-up of a neutrino multiplet

Grid Laboratory of Wisconsin (GLOW) grid infrastructure at the University of Wisconsin - Madison, the Open Science Grid (OSG) grid infrastructure; US Department of Energy, and National Energy Research Scientific Computing Center, the Louisiana Optical Network Initiative (LONI) grid computing resources; Natural Sciences and Engineering Research Council of Canada, WestGrid and Compute/Calcul Canada; Swedish Research Council, Swedish Polar Research Secretariat, Swedish National Infrastructure for Computing (SNIC), and Knut and Alice Wallenberg Foundation, Sweden; German Ministry for Education and Research (BMBF), Deutsche Forschungsgemeinschaft (DFG), Helmholtz Alliance for Astroparticle Physics (HAP), Research Department of Plasmas with Complex Interactions (Bochum), Germany; Fund for Scientific Research (FNRSFWO), FWO Odysseus programme, Flanders Institute to encourage scientific and technological research in industry (IWT), Belgian Federal Science Policy Office (Belspo); University of Oxford, United Kingdom; Marsden Fund, New Zealand; Australian Research Council; Japan Society for Promotion of Science (JSPS); the Swiss National Science Foundation (SNSF), Switzerland; National Research Foundation of Korea (NRF); Villum Fonden, Danish National Research Foundation (DNRF), Denmark This work made use of data supplied by the UK Swift Science Data Centre at the University of Leicester. Funding for the Swift project in the UK is provided by the UK Space Agency. Part of this work was facilitated by the GROWTH project, a partnership in international research and education, NSF PIRE Grant No. 1545949. ASAS-SN is supported by NSF grant AST-1515927. Development of ASAS-SN has been supported by NSF grant AST-0908816, the Center for Cosmology and AstroParticle Physics at the Ohio State University, the Mt. Cuba Astronomical Foundation, and by George Skestos. The Fermi LAT Collaboration acknowledges generous ongoing support from a number of agencies and institutes that have supported both the development and the operation of the LAT as well as scientific data analysis. These include the National Aeronautics and Space Administration and the Department of Energy in the United States, the Commissariat à l'Energie Atomique and the Centre National de la Recherche Scientifique/Institut National de Physique Nucléaire et de Physique des Particules in France, the Agenzia Spaziale Italiana and the Istituto Nazionale di Fisica Nucleare in Italy, the Ministry of Education, Culture, Sports, Science and Technology (MEXT), high-energy Accelerator Research Organization (KEK) and Japan Aerospace Exploration Agency (JAXA) in Japan and the K. A. Wallenberg Foundation, the Swedish Research Council and the Swedish National Space Board in Sweden. Additional support for science analysis during the operations phase is gratefully acknowledged from the Istituto Nazionale di Astrofisica in Italy and the Centre National d'Études Spatiales in France. The HAWC Collaboration acknowledges the support from: the US National Science Foundation (NSF); the US Department of Energy Office of HighEnergy Physics; the Laboratory Directed Research and Development (LDRD) program of Los Alamos National Laboratory; Consejo Nacional de Ciencia y Tecnología (CONACyT), México (grants 271051, 232656, 260378, 179588, 239762, 254964, 271737, 258865, 243290, 132197), Laboratorio Nacional HAWC de rayos gamma; L'OREAL Fellowship for Women in Science 2014 Red HAWC, México; DGAPA-UNAM (grants IG100317, IN111315, IN1117163, IA102715, 109916, IA102917); VIEP-BUAP; PIFI 2012, 2013, PROFOCIE 2014, 2015; the University of Wisconsin Alumni Research Foundation; the In stitute of Geophysics, Planetary Physics, and Signatures at Los Alamos National Laboratory; Polish Science Centre grant DEC-2014/13/B/ST9/945; Coordinación de la Investigación Científica de la Universidad Michoacana. We thank Luciano Díaz and Eduardo Murrieta for technical support of the HAWC detector. Support for I. Arcavi was provided by NASA through the Einstein Fellowship Program, grant PF6-170148. D. A. Howell, C. McCully, and G. Hosseinzadeh are supported by NSF-1313484. This work makes use of observations from the LCO network. VERITAS research is supported by grants from the U.S. Department of Energy Office of Science, the U.S. National Science Foundation and the Smithsonian Institution, and by NSERC in Canada. VERITAS acknowledges the excellent work of the technical support staff at the Fred Lawrence Whipple Observatory and at the collaborating institutions in the construction and operation of the instrument. E. O. Ofek and A. Gal-Yam acknowledge a Minerva grant.

\section{References}

Aartsen, M. G., Abbasi, R., Abdou, Y., et al. 2013, Science, 342, 1242856 Aartsen, M. G., Ackermann, M., Adams, J., et al. 2014, ApJ, 796, 109 Aartsen, M. G., Abraham, K., Ackermann, M., et al. 2015a, ApJ, 809, 98 Aartsen, M. G., Abraham, K., Ackermann, M., et al. 2015b, ApJ, 811, 52 Aartsen, M. G., Ackermann, M., Adams, J., et al. 2015c, ApJ, 805, L5 Aartsen, M. G., Abraham, K., Ackermann, M., et al. 2016a, ApJ, 833, 3 Aartsen, M. G., Abraham, K., Ackermann, M., et al. 2016b, J. Inst., 11, P11009 Aartsen, M. G., Abraham, K., Ackermann, M., et al. 2016c, ApJ, 824, 115 Aartsen, M. G., Abraham, K., Ackermann, M., et al. 2017a, ApJ, 835, 45 Aartsen, M. G., Abraham, K., Ackermann, M., et al. 2017b, ApJ, 835, 151 Aartsen, M. G., Ackermann, M., Adams, J., et al. 2017c, J. Inst., 12, P03012
Aartsen, M. G., Ackermann, M., Adams, J., et al. 2017d, Astropart. Phys., 92, 30 Abbasi, R., Ackermann, M., Adams, J., et al. 2009, Nucl. Inst. Meth. Phys. Res. A, 601, 294

Abbasi, R., Abdou, Y., Abu-Zayyad, T., et al. 2010a, Nucl. Inst. Meth. Phys. Res. A, 618, 139

Abbasi, R., Abdou, Y., Abu-Zayyad, T., et al. 2010b, ApJ, 718, L194 Abbasi, R., Abdou, Y., Abu-Zayyad, T., et al. 2012a, Nature, 484, 351 Abbasi, R., Abdou, Y., Abu-Zayyad, T., et al. 2012b, A\&A, 539, A60 Abdo, A. A., Ackermann, M., Ajello, M., et al. 2009, ApJS, 183, 46 Abeysekara, A. U., Alfaro, R., Alvarez, C., et al. 2016, ApJ, 817, 3 Abeysekara, A. U., Albert, A., Alfaro, R., et al. 2017a, ApJ, 843, 40 Abeysekara, A. U., Albert, A., Alfaro, R., et al. 2017b, ApJ, 843, 39 Acero, F., Ackermann, M., Ajello, M., et al. 2015, ApJS, 218, 23 Achterberg, A., Ackermann, M., Adams, J., et al. 2006, Astropart. Phys., 26, 155 Ackermann, M., Ajello, M., Baldini, L., et al. 2012, ApJ, 754, 121 Ackermann, M., Ajello, M., Albert, A., et al. 2013a, ApJ, 771, 57 Ackermann, M., Ajello, M., Asano, K., et al. 2013b, ApJS, 209, 11 Ackermann, M., Ajello, M., Atwood, W. B., et al. 2015, ApJ, 810, 14 Adrián-Martínez, S., Ageron, M., Albert, A., et al. 2016, J. Cosmol. Astropart. Phys., 2, 062

Ageron, M., Aguilar, J. A., Al Samarai, I., et al. 2012, Astropart. Phys., 35, 530 Agüeros, M. A., Anderson, S. F., Covey, K. R., et al. 2009, ApJS, 181, 444 Ahlers, M., \& Halzen, F. 2014, Phys. Rev. D, 90, 043005

Ahrens, J., Bai, X., Bay, R., et al. 2004, Nucl. Inst. Meth. Phys. Res. A, 524, 169 Aird, J., Coil, A. L., Georgakakis, A., et al. 2015, MNRAS, 451, 1892 Aleksić, J., Ansoldi, S., Antonelli, L. A., et al. 2016, Astropart. Phys., 72, 76 Aliu, E., Archambault, S., Arlen, T., et al. 2012, ApJ, 759, 102 Anderhub, H., Backes, M., Biland, A., et al. 2013, J. Inst., 8, P06008 Atwood, W. B., Abdo, A. A., Ackermann, M., et al. 2009, ApJ, 697, 1071 Baerwald, P., Bustamante, M., \& Winter, W. 2015, Astropart. Phys., 62, 66 Barthelmy, S. D., Barbier, L. M., Cummings, J. R., et al. 2005, Space Sci. Rev., 120, 143

Baumgartner, W. H., Tueller, J., Markwardt, C. B., et al. 2013, ApJS, 207, 19 Bellm, E. 2014, in The Third Hot-wiring the Transient Universe Workshop, eds.

P. R. Wozniak, M. J. Graham, A. A. Mahabal, \& R. Seaman, 27

Berge, D., Funk, S., \& Hinton, J. 2007, A\&A, 466, 1219

Bertin, E., \& Arnouts, S. 1996, A\&AS, 117, 393

Blaufuss, E. 2016, GRB Coordinates Network, 19363,

Brown, T. M., Baliber, N., Bianco, F. B., et al. 2013, PASP, 125, 1031

Burlon, D., Ajello, M., Greiner, J., et al. 2011, ApJ, 728, 58

Bustamante, M., Baerwald, P., Murase, K., \& Winter, W. 2015, Nature Commun., 6,6783

Cano, Z. 2014, ApJ, 794, 121

Cano, Z., Wang, S.-Q., Dai, Z.-G., \& Wu, X.-F. 2017, Adv. Astron., 2017, 8929054

Cappellaro, E., Botticella, M. T., Pignata, G., et al. 2015, A\&A, 584, A62

Cenko, S. B., Kulkarni, S. R., Horesh, A., et al. 2013, ApJ, 769, 130

Cenko, S. B., Urban, A. L., Perley, D. A., et al. 2015, ApJ, 803, L24

Dermer, C. D., \& Giebels, B. 2016, Comptes Rendus Physique, 17, 594

Dornic, D., Basa, S., Evans, P. A., et al. 2015, ATel, 7987

Evans, P. A., Willingale, R., Osborne, J. P., et al. 2010, A\&A, 519, A102

Evans, P. A., Osborne, J. P., Beardmore, A. P., et al. 2014, ApJS, 210, 8

Evans, P. A., Osborne, J. P., Kennea, J. A., et al. 2015, MNRAS, 448, 2210

Evans, P. A., Osborne, J. P., Kennea, J. A., et al. 2016, MNRAS, 455, 1522

Farrar, G. R., \& Piran, T. 2014, ArXiv e-prints [arXiv: 1411.0704]

Fraija, N. 2014, MNRAS, 437, 2187

Galama, T. J., Vreeswijk, P. M., van Paradijs, J., et al. 1998, Nature, 395, 670

Gehrels, N., Chincarini, G., Giommi, P., et al. 2004, ApJ, 611, 1005

Ghirlanda, G., Salvaterra, R., Campana, S., et al. 2015, A\&A, 578, A71

Gorbovskoy, E. S., Lipunov, V. M., Kornilov, V. G., et al. 2013, Astron. Rep., 57,233

Górski, K. M., Hivon, E., Banday, A. J., et al. 2005, ApJ, 622, 759

Gruber, D., Goldstein, A., Weller von Ahlefeld, V., et al. 2014, ApJS, 211, 12

Healey, S. E., Romani, R. W., Taylor, G. B., et al. 2007, ApJS, 171, 61

Henden, A. A., Levine, S., Terrell, D., \& Welch, D. L. 2015, in Am. Astron. Soc. Meeting Abstracts, 225, 336.16

Hurley, K., Golenetskii, S., Aptekar, R., et al. 2010, in AIP Conf. Ser., 1279, eds.

N. Kawai, \& S. Nagataki, 330

Kadler, M., Krauß, F., Mannheim, K., et al. 2016, Nature Phys., 12, 807

Kann, D. A., Klose, S., Zhang, B., et al. 2010, ApJ, 720, 1513

Kann, D. A., Klose, S., Zhang, B., et al. 2011, ApJ, 734, 96

Kann, D. A., Schady, P., Olivares E., F., et al. 2017, A\&A, submitted [arXiv: 1606.06791]

Kathirgamaraju, A., Barniol Duran, R., \& Giannios, D. 2016, MNRAS, 461, 1568

Katz, B., Sapir, N., \& Waxman, E. 2011, ArXiv e-prints [arXiv: 1106. 1898]

Kintscher, T. 2016, in Eur. Phys. J. Web Conf., 116, 10002

Komossa, S. 2015, J. High Energy Astrophys., 7, 148 
Kornilov, V. G., Lipunov, V. M., Gorbovskoy, E. S., et al. 2012, Exp. Astron., 33, 173

Kowalski, M. 2015, J. Phys. Conf. Ser., 632, 012039

Kraft, R. P., Burrows, D. N., \& Nousek, J. A. 1991, ApJ, 374, 344

Krühler, T., Malesani, D., Fynbo, J. P. U., et al. 2015, A\&A, 581, A125

Law, N. M., Kulkarni, S. R., Dekany, R. G., et al. 2009, PASP, 121, 1395

Liang, E., Zhang, B., Virgili, F., \& Dai, Z. G. 2007, ApJ, 662, 1111

Lipunov, V., Kornilov, V., Gorbovskoy, E., et al. 2010, Adv. Astron., 2010, 349171

Lipunov, V. M., Gorosabel, J., Pruzhinskaya, M. V., et al. 2016, MNRAS, 455 712

Madau, P., \& Dickinson, M. 2014, ARA\&A, 52, 415

Markwardt, C. B., Tueller, J., Skinner, G. K., et al. 2005, ApJ, 633, L77

Meegan, C., Lichti, G., Bhat, P. N., et al. 2009, ApJ, 702, 791

Mészáros, P. 2015, ArXiv e-prints [arXiv: 1511.01396]

Murase, K. 2015, ArXiv e-prints [arXiv: 1511.01590]

Murase, K., \& Ioka, K. 2013, Phys. Rev. Lett., 111, 121102

Murase, K., \& Waxman, E. 2016, Phys. Rev. D, 94, 103006

Murase, K., Thompson, T. A., Lacki, B. C., \& Beacom, J. F. 2011, Phys. Rev. D 84, 043003

Murase, K., Thompson, T. A., \& Ofek, E. O. 2014, MNRAS, 440, 2528

Nilsson, K., Pursimo, T., Villforth, C., et al. 2012, A\&A, 547, A1

Padovani, P., Resconi, E., Giommi, P., Arsioli, B., \& Chang, Y. L. 2016 MNRAS, 457, 3582

Park, N. 2015, Pos(ICRC2015)771

Pfeffer, D. N., Kovetz, E. D., \& Kamionkowski, M. 2017, MNRAS, 466, 2922

Rau, A., Kulkarni, S. R., Law, N. M., et al. 2009, PASP, 121, 1334

Salvaterra, R., Campana, S., Vergani, S. D., et al. 2012, ApJ, 749, 68

Schoenen, S., \& Raedel, L. 2015, ATel, 7856

Senno, N., Murase, K., \& Mészáros, P. 2016, Phys. Rev. D, 93, 083003

Shappee, B., Prieto, J., Stanek, K. Z., et al. 2014, in Am. Astron. Soc. Meeting Abstracts, 223, 236.03

Smartt, S. J., Chambers, K., Smith, K., \& Hub, M. 2015, ATel, 7992

Strolger, L.-G., Dahlen, T., Rodney, S. A., et al. 2015, ApJ, 813, 93

Strotjohann, N. L., Saxton, R. D., Starling, R. L. C., et al. 2016, A\&A, 592, A74

Sun, H., Zhang, B., \& Gao, H. 2017, ApJ, 835, 7

Tamborra, I., \& Ando, S. 2016, Phys. Rev. D, 93, 053010

Tamborra, I., Ando, S., \& Murase, K. 2014, J. Cosmol. Astropart. Phys., 9, 043

Tueller, J., Mushotzky, R. F., Barthelmy, S., et al. 2008, ApJ, 681, 113

Tueller, J., Baumgartner, W. H., Markwardt, C. B., et al. 2010, ApJS, 186, 378

Valenti, S., Howell, D. A., Stritzinger, M. D., et al. 2016, MNRAS, 459, 3939

Voges, W., \& Boller, T. 1999, Mem. Soc. Astron. Ital., 70, 839

Wanderman, D., \& Piran, T. 2010, MNRAS, 406, 1944

Wang, X.-Y., \& Liu, R.-Y. 2016, Phys. Rev. D, 93, 083005

Waxman, E. 2015, ArXiv e-prints [arXiv: 1511.00815]

Wills, B. J., \& Browne, I. W. A. 1986, ApJ, 302, 56

Wright, N. J., Drake, J. J., \& Civano, F. 2010, ApJ, 725, 480

Zou, Y. C., Wu, X. F., \& Dai, Z. G. 2007, A\&A, 461, 115

1 III. Physikalisches Institut, RWTH Aachen University, 52056 Aachen, Germany

2 Department of Physics, University of Adelaide, Adelaide 5005, Australia

3 Dept of Physics and Astronomy, University of New Mexico, Albuquerque, NM 87131, USA

4 Fred Lawrence Whipple Observatory, Harvard-Smithsonian Center for Astrophysics, Amado, AZ 85645, USA

5 Department of Physics and Astronomy, Iowa State University, Ames, IA 50011, USA

6 Dept. of Physics and Astronomy, University of Alaska Anchorage, 3211 Providence Dr., Anchorage, AK 99508, USA

7 CTSPS, Clark-Atlanta University, Atlanta, GA 30314, USA

8 School of Physics and Center for Relativistic Astrophysics, Georgia Institute of Technology, Atlanta, GA 30332, USA

9 Dept. of Physics, Southern University, Baton Rouge, LA 70813, USA

10 Department of Physics and Center for Astrophysics, Tsinghua University, Beijing 100084, PR China

11 Dept. of Physics, University of California, Berkeley, CA 94720, USA

12 Lawrence Berkeley National Laboratory, Berkeley, CA 94720, USA
13 Institut für Physik, Humboldt-Universität zu Berlin, 12489 Berlin, Germany

14 Fakultät für Physik \& Astronomie, Ruhr-Universität Bochum, 44780 Bochum, Germany

15 Physikalisches Institut, Universität Bonn, Nussallee 12, 53115 Bonn, Germany

16 Université Libre de Bruxelles, Science Faculty CP230, 1050 Brussels, Belgium

17 Vrije Universiteit Brussel, Dienst ELEM, 1050 Brussels, Belgium

18 Dept. of Physics, Massachusetts Institute of Technology, Cambridge, MA 02139, USA

${ }^{19}$ Instituto de Astronomía, Universidad Nacional Autónoma de México, Ciudad de Mexico, Mexico

${ }^{20}$ Instituto de Física, Universidad Nacional Autónoma de México, Ciudad de Mexico, Mexico

${ }^{21}$ Instituto de Geofísica, Universidad Nacional Autónoma de México, Ciudad de Mexico, Mexico

22 Centro de Investigación en Computación, Instituto Politécnico Nacional, México City, Mexico

23 Physics Department, Centro de Investigacion y de Estudios Avanzados del IPN, Mexico City, DF, Mexico

${ }^{24}$ Instituto de Ciencias Nucleares, Universidad Nacional Autónoma de Mexico, Ciudad de Mexico, Mexico

25 Universidad Autónoma de Chiapas, Tuxtla Gutiérrez, Chiapas, Mexico

26 Dept. of Physics and Institute for Global Prominent Research, Chiba University, Chiba 263-8522, Japan

27 Enrico Fermi Institute, University of Chicago, Chicago, IL 60637, USA

28 Dept. of Physics and Astronomy, University of Canterbury, Private Bag 4800, Christchurch, New Zealand

29 Dept. of Physics, University of Maryland, College Park, MD 20742, USA

30 Dept. of Physics and Center for Cosmology and AstroParticle Physics, Ohio State University, Columbus, OH 43210, USA

31 Dept. of Astronomy, Ohio State University, Columbus, OH 43210, USA

32 Niels Bohr Institute, University of Copenhagen, 2100 Copenhagen, Denmark

33 Department of Physical Sciences, Cork Institute of Technology, Bishopstown, Cork, Ireland

34 Department of Physics, University of California, 1 Shields Ave, Davis, CA 95616-5270, USA

35 Dept. of Physics, TU Dortmund University, 44221 Dortmund, Germany

36 School of Physics, University College Dublin, Belfield, Dublin 4, Ireland

37 Dept. of Physics and Astronomy, Michigan State University, East Lansing, MI 48824, USA

38 Dept. of Physics, University of Alberta, Edmonton, Alberta, T6G 2E1, Canada

39 Einstein Fellow

40 Erlangen Centre for Astroparticle Physics, Friedrich-AlexanderUniversität Erlangen-Nürnberg, 91058 Erlangen, Germany

41 School of Physics, Astronomy, and Computational Sciences, George Mason University, Fairfax, VA, USA

42 School of Physics, National University of Ireland Galway, University Road, Galway, Ireland

43 Département de physique nucléaire et corpusculaire, Université de Genève, 1211 Genève, Switzerland

44 Dept. of Physics and Astronomy, University of Gent, 9000 Gent, Belgium

45 Las Cumbres Observatory, 6740 Cortona Dr, Ste 102, Goleta, CA 93117-5575, USA

46 Instituto de Astrofísica de Andalucía (IAA-CSIC), Glorieta de la Astronomía s/n, 18008 Granada, Spain

47 NASA Goddard Space Flight Center, 8800 Greenbelt Road, Greenbelt, MD 20771, USA 
48 Department of Physics and Astronomy, DePauw University, Greencastle, IN 46135-0037, USA

49 Departamento de Física, Centro Universitario de Ciencias Exactase Ingenierias, Universidad de Guadalajara, Guadalajara, Mexico

50 Department of Physics, California State University - East Bay, Hayward, CA 94542, USA

51 Max-Planck Institute for Nuclear Physics, 69117 Heidelberg, Germany

52 Universidad Politecnica de Pachuca, Pachuca, Hgo, Mexico

53 Department of Physics, Stevens Institute of Technology, Hoboken, NJ 07030, USA

54 Department of Physics, Michigan Technological University, Houghton, MI 49931, USA

55 Hubble and Carnegie-Princeton Fellow

56 NASA Marshall Space Flight Center, Astrophysics Office, Huntsville, AL 35812, USA

57 Department of Physics and Astronomy, University of Iowa, Van Allen Hall, Iowa City, IA 52242, USA

58 Institute of Applied Physics, Irkutsk State University 20, Gagarin Blvd, Irkutsk, 664003, Russia

59 Dept. of Physics and Astronomy, University of California, Irvine, CA 92697, USA

60 Kislovodsk Solar Station of Main Pulkovo Observatory, PO Box 45, ul. Gagarina 100, Kislovodsk 357700, Russia

61 Instytut Fizyki Jadrowej im Henryka Niewodniczanskiego Polskiej Akademii Nauk, IFJ-PAN, 31-342 Krakow, Poland

62 Dept. of Physics and Astronomy, University of Kansas, Lawrence, KS 66045, USA

${ }^{63}$ University of Leicester, X-ray and Observational Astronomy Research Group, Leicester Institute for Space and Earth Observation, Dept. of Physics \& Astronomy, University Road, Leicester, LE1 7RH, UK

${ }^{64}$ Physics Division, Los Alamos National Laboratory, Los Alamos, NM 87545, USA

65 Department of Physics and Astronomy, University of California, Los Angeles, CA 90095, USA

66 Dept. of Astronomy, University of Wisconsin, Madison, WI 53706, USA

67 Dept. of Physics and Wisconsin IceCube Particle Astrophysics Center, University of Wisconsin, Madison, WI 53706, USA

68 Institute of Physics, University of Mainz, Staudinger Weg 7, 55099 Mainz, Germany

69 Department of Physics, Marquette University, Milwaukee, WI, 53201, USA

70 School of Physics and Astronomy, University of Minnesota, Minneapolis, MN 55455, USA

71 Université de Mons, 7000 Mons, Belgium

72 Physics Department, McGill University, Montreal, QC H3A 2T8, Canada

73 Universidad Michoacana de San Nicolás de Hidalgo, Morelia, Mexico

74 Lomonosov Moscow State University, Physics Department, Leninskie gory, GSP-1, Moscow 119991, Russia

75 Lomonosov Moscow State University, Sternberg Astronomical Institute, Universitetsky Prospekt 13, Moscow 119192, Russia

76 Physik-department, Technische Universität München, 85748 Garching, Germany

77 Institut für Kernphysik, Westfälische Wilhelms-Universität Münster, 48149 Münster, Germany

78 Bartol Research Institute and Dept. of Physics and Astronomy, University of Delaware, Newark, DE 19716, USA

79 Dept. of Physics, Yale University, New Haven, CT 06520, USA

${ }^{80}$ Physics Department, Columbia University, New York, NY 10027, USA

81 Department of Physics and Astronomy, Barnard College, Columbia University, NY 10027, USA

82 Dept. of Physics, University of Oxford, 1 Keble Road, Oxford OX1 3NP, UK
83 Universidad Autónoma del Estado de Hidalgo, Pachuca, Mexico

${ }^{84}$ Carnegie Observatories, 813 Santa Barbara Street, Pasadena, CA 91101, USA

85 Cahill Center for Astronomy and Astrophysics, California Institute of Technology, Pasadena, CA 91125, USA

86 Dept. of Physics, Drexel University, 3141 Chestnut Street, Philadelphia, PA 19104, USA

87 Institute of Physics and Astronomy, University of Potsdam, 14476 Potsdam-Golm, Germany

88 Instituto Nacional de Astrofísica, Óptica y Electrónica, Puebla, Mexico

89 Facultad de Ciencias Físico Matemáticas, Benemérita Universidad Autónoma de Puebla, Puebla, Mexico

90 Physics Department, South Dakota School of Mines and Technology, Rapid City, SD 57701, USA

91 Department of Particle Physics \& Astrophysics, Weizmann Institute of Science, Rehovot 7610001, Israel

92 Dept. of Physics, University of Wisconsin, River Falls, WI 54022, USA

93 Dept. of Physics and Astronomy, University of Rochester, Rochester, NY 14627, USA

94 Department of Physics and Astronomy, University of Utah, Salt Lake City, UT 84112, USA

95 Department of Physics, University of California, Santa Barbara, CA 93106-9530, USA

96 Kavli Institute for Theoretical Physics, University of California, Santa Barbara, CA 93106-4030, USA

97 Santa Cruz Institute for Particle Physics and Department of Physics, University of California, Santa Cruz, CA 95064, USA

98 Nucleo de Astronomia de la Facultad de Ingenieria, Universidad Diego Portales, Av. Ejercito 441, Santiago, Chile

99 Millennium Institute of Astrophysics, Santiago, Chile

100 Department of Physics, Washington University, St. Louis, MO 63130, USA

101 Oskar Klein Centre and Dept. of Physics, Stockholm University, 10691 Stockholm, Sweden

102 Dept. of Physics and Astronomy, Stony Brook University, Stony Brook, NY 11794-3800, USA

103 Dept. of Physics, Sungkyunkwan University, Suwon 440-746, Korea

104 Thüringer Landessternwarte Tautenburg, Sternwarte 5, 07778 Tautenburg, Germany

105 Instituto de Astrofísica de Canarias, Observatorio del Teide C/via Lactea, s/n, 38205 La Laguna, Tenerife, Spain

106 Earthquake Research Institute, University of Tokyo, Bunkyo, Tokyo 113-0032, Japan

107 Dept. of Physics, University of Toronto, Toronto, Ontario M5S 1A7, Canada

108 Dept. of Physics and Astronomy, University of Alabama, Tuscaloosa, AL 35487, USA

109 Dept. of Astronomy and Astrophysics, Pennsylvania State, University, University Park, PA 16802, USA

110 Dept. of Physics, Pennsylvania State University, University Park, PA 16802, USA

111 Center for Particle \& Gravitational Astrophysics, Institute for Gravitation and the Cosmos, Pennsylvania State University, University Park, PA 16802, USA

112 Center for Theoretical \& Observational Cosmology, Institute for Gravitation and the Cosmos, Pennsylvania State University, University Park, PA 16802, USA

113 Dept. of Physics and Astronomy, Uppsala University, Box 516, 75120 Uppsala, Sweden

114 Department of Physics and Astronomy, Purdue University, West Lafayette, IN 47907, USA

115 Dept. of Physics, University of Wuppertal, 42119 Wuppertal, Germany

116 DESY, 15735 Zeuthen, Germany 


\section{Appendix A: The nature of the X-ray source X6}

As described in Sect. 4.2.2, we detected a highly variable X-ray source (see Fig. A.1) without an obvious optical counterpart. The first Swift detection of X6 has a probability of a spurious detection of $<0.3 \%$ (Evans et al. 2014). Since it was later re-detected with high confidence, we consider X6 a genuine astrophysical source.

The X-ray light curve of X6 is consistent with a $t^{-0.5}$ decay over a period of 5 months. During this time, its X-ray flux in the 0.3 to $10.0 \mathrm{keV}$ energy range fades by a factor of nine. As an aside, we note that the source appears to exhibit variability during the third XRT observation, where 9 out of 11 counts are detected during the first $45 \%$ of the exposure time. However, closer investigation revealed that this was due to the source being placed near a bad column on the detector, which leads to lost counts during the second half of the exposure. The flux estimate for the source takes this loss of exposure into account.

The probability of detecting a serendipitous X-ray source at the flux level of X6 is 5\% when considering the covered area as well as the exposure time of the tiled XRT observation (Evans et al. 2015). Voges \& Boller (1999) systematically studied the variability of X-ray sources detected by ROSAT in the $0.07-2.4 \mathrm{keV}$ energy range. They find that $9 \%$ of the sources are variable by a factor of more than three. Out of those sources $57 \%$ are unidentified, $30 \%$ are stars and the remaining $13 \%$ are extragalactic sources; mostly AGN. Only $0.7 \%$ of the sources in their sample are variable by a factor of 10 or more. The detection of $\mathrm{X} 6$ is hence unexpected.

We identified two possible scenarios that are consistent with all obtained observations. The X-rays could be emitted by a distant and obscured highly variable AGN. Alternatively they could be associated with one of two nearby stars, S1 or S2 (see Fig. A.2), or an X-ray bright binary companion of one the stars. Neither scenario yields a detectable neutrino flux on Earth.

\section{A.1. A distant active galactic nucleus}

The faint object, O3, is the only detected source within the $90 \%$ error circle of X6 (compare Fig. A.2 and Table A.1). Since we do not have a spectrum or additional photometric points we do not know whether it is a star, a compact galaxy, or an AGN. An AGN could easily account for the detected X-ray flux even if it is located at a high redshift ( $z \gtrsim 1$; see e.g., Aird et al. 2015). It is also possible that $\mathrm{O} 3$ is an unrelated object and that an even fainter AGN is located within the error circle of X6.

An AGN can be faint in the optical if the accretion disk and jet, if present, are obscured by dust. If it is located at a high redshift its host galaxy may not be detectable either. The absence of a bright optical counterpart is therefore not unusual, but it does indicate that the AGN likely is not close-by.

AGN typically have variable X-ray luminosities due to perturbations in their accretion disk. However, large amplitude variability, as observed for X6, is only detected for a few percent of all AGN (Strotjohann et al. 2016). Such bright X-ray flares can, for example, be caused by changing jet activity in blazars. No gamma-ray emission is detected by the Fermi LAT, VERITAS, or HAWC (compare Sect. 4.3) and no known radio source is consistent with the position of X6. So there is no further evidence for a flaring blazar and if a jet is present, it does not emit a strong flux of $\mathrm{GeV}$ or $\mathrm{TeV}$ photons.

Even though blazars are promising candidates for the emission of high-energy neutrinos (see e.g., Padovani et al. 2016, and references therein), it seems unlikely that a rather faint X-ray

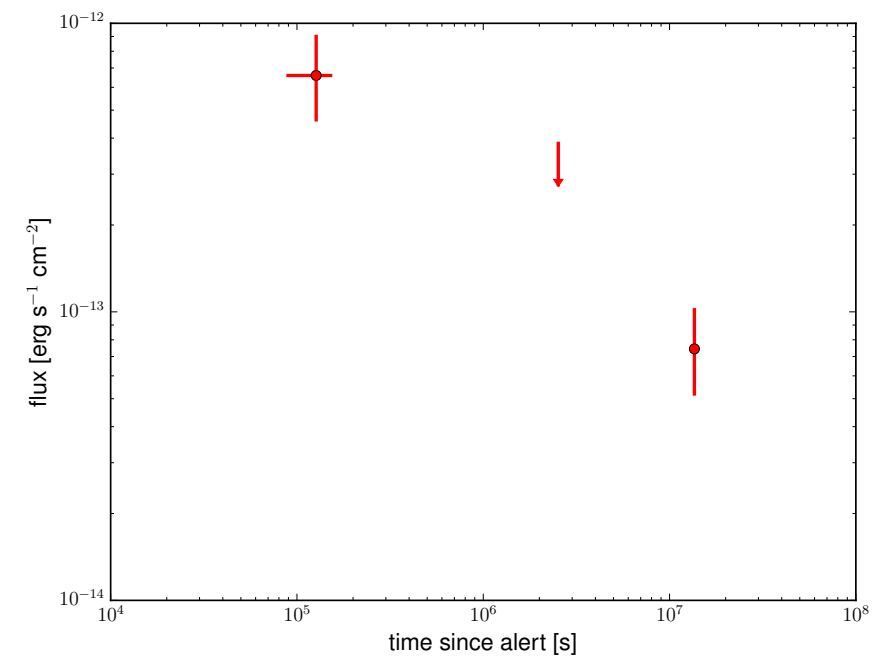

Fig. A.1. XRT light curve of X6 in the $0.3-10 \mathrm{keV}$ range. The error bars are at the $1 \sigma$ level and the upper limit is at $3 \sigma$ confidence.

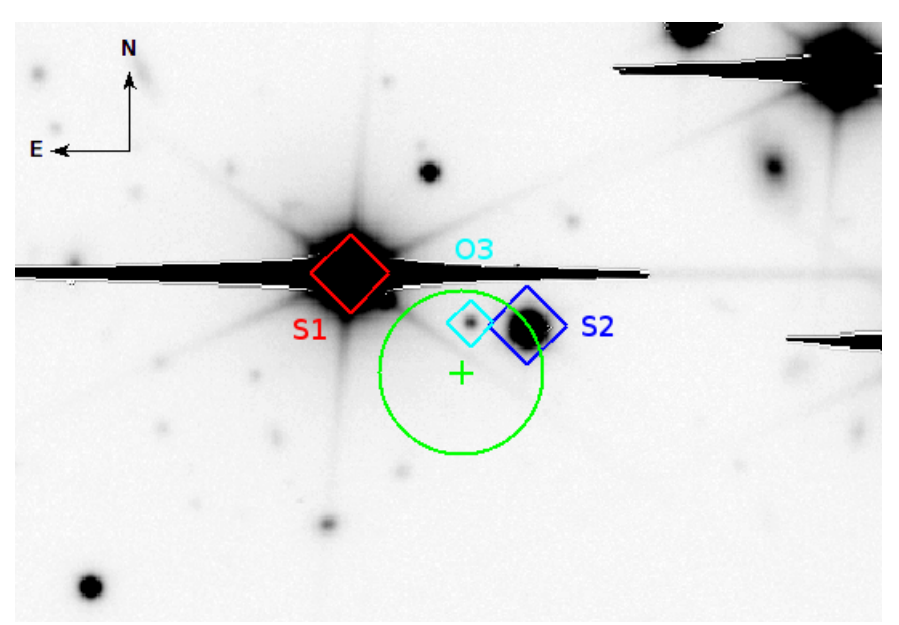

Fig. A.2. Keck/LRIS image. Shown in green are the position of X6 and the $90 \%$ error circle which has a radius of $6.2^{\prime \prime}$. Three potential optical counterparts are marked with diamonds: $\mathrm{S} 1$ in red, S2 in blue and O3 in cyan (see Table A.1 for details). While S1 and S2 are Sun-like stars (see Fig. A.3), the nature of $\mathrm{O} 3$ is unknown.

source that is not detected at higher energies emits a strong neutrino flux. We therefore do not consider this AGN candidate a possible counterpart for the detected neutrinos.

\section{A.2. Stellar $X$-ray flares}

In addition to $\mathrm{O} 3$, the stars $\mathrm{S} 1$ and $\mathrm{S} 2$, are located close to the $90 \%$ error circle of X6 as shown in Fig. A.2 and Table A.1. Especially S2 is just at the edge of the error circle and has a reasonable chance to be associated with X6. Optical spectra taken with LCO are shown in Fig. A.3. The hydrogen absorption lines at redshift zero show that both sources are stars. The temperature of $\mathrm{S} 2$ is very similar to the Sun (class G2) while $\mathrm{S} 1$ has a higher temperature. It could either be a hot $\mathrm{G}$ star or a low-temperature F $\operatorname{star}^{19}$.

A rough estimate can show whether $\mathrm{S} 2$ can account for the detected X-ray flux. Assuming that the star has solar luminosity

\footnotetext{
19 Standard spectra for comparison can be found at http://classic. sdss.org/dr5/algorithms/spectemplates/
} 
Table A.1. Possible optical counterparts of X6.

\begin{tabular}{lllllll}
\hline \hline Name & Object type & $\begin{array}{l}\text { RA } \\
\left({ }^{\circ}\right)\end{array}$ & $\begin{array}{l}\text { Dec } \\
\left({ }^{\circ}\right)\end{array}$ & $\begin{array}{l}\text { Ang. Sep. from X6 } \\
\left({ }^{\prime \prime}\right)\end{array}$ & $\begin{array}{l}\text { Distance } \\
(\mathrm{pc})\end{array}$ & $\begin{array}{l}R \text { band magnitude } \\
(\mathrm{mag})\end{array}$ \\
\hline S1 & F or G star & 25.01375 & +39.60553 & 11.6 & $\sim 510$ & 13.0 \\
S2 & G star & 25.00892 & +39.60431 & 6.2 & $\sim 1500$ & 15.8 \\
O3 & unknown & 25.01044 & +39.60440 & 3.9 & unknown & 20.7 \\
\hline
\end{tabular}

Notes. The locations of the three objects are shown in Fig. A.2 and the spectra of S1 and S2 are presented in Fig. A.3. The magnitudes of S1 and S2 have been measured from PTF images and the one of O3 is from the Keck/LRIS image. All magnitudes are approximate because the point spread functions of the three objects overlap.

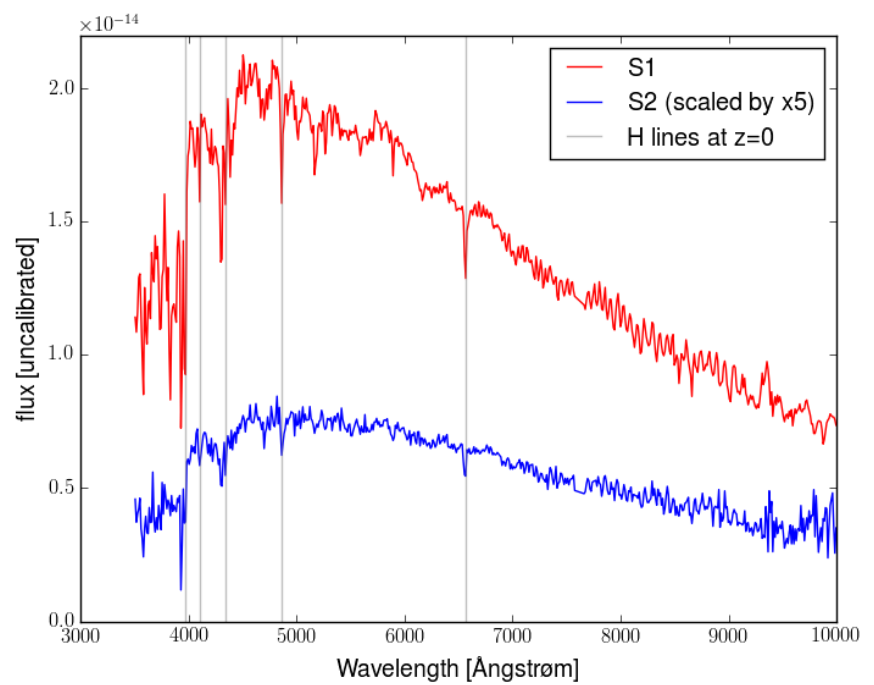

Fig. A.3. LCO spectra of S1 and S2 (compare Fig. A.2). Hydrogen absorption lines show that they are $\mathrm{F}$ or $\mathrm{G}$ stars in our Galaxy. Telluric bands at $6870 \AA$ and $7600 \AA$ were removed from the spectra.

we estimate its distance to be $\sim 1500 \mathrm{pc}$. Based on this distance the X-ray luminosity is $\sim 10^{32} \mathrm{erg} \mathrm{s}^{-1}$, which is a factor of $10^{4}$ brighter than the flaring Sun. Assuming again solar luminosity $\mathrm{S} 1$ is located at $\sim 500 \mathrm{pc}$ and would have to emit an X-ray luminosity of $\sim 10^{31} \mathrm{erg} \mathrm{s}^{-1}$ to account for the detected X-ray flux. In the samples presented by Agüeros et al. (2009) and Wright et al. (2010) less than one percent of the stars detected in X-rays reach luminosities above $10^{31} \mathrm{erg} \mathrm{s}^{-1}$ and only $\sim 10$ such stars have ever been detected. If S1 or S2 is the source of the X-rays, the star underwent an extreme flare.

Extreme stellar X-ray flares can be emitted by close or active binary systems (see e.g., Wright et al. 2010). It is possible S1 or S2 has a binary partner that is too faint to be detectable in the optical spectra in Fig. A.3. The spectra do not show Balmer emission lines thus there is no evidence for an accretion disk. However, a close binary without mass transfer would be consistent with our observations. To search for evidence for a binary system we analyze the forced photometry light curve of S2 which consists of $185 \mathrm{~g}$ band PTF images acquired over more than three years. While there is evidence for variability at a low level of $0.05 \mathrm{mag}_{g}$, no significant period was detected. The optical light curve hence does not provide evidence for a binary partner, but neither can we rule out its presence. We cannot repeat this analysis for $\mathrm{S} 1$ which is saturated in most PTF observations. $\mathrm{O} 3$ is not detected in individual or stacked PTF observations and we hence do not know whether or not it is variable in the optical.

Another possibility is that $\mathrm{O} 3$ is a nearby faint star (e.g., an $\mathrm{M}$ dwarf) that undergoes a strong X-ray flare or it could be an $\mathrm{X}$-ray binary. Due to the lack of an optical spectrum we cannot verify this scenario.

\section{A.3. Conclusion}

We detected a highly variable but faint X-ray source which could be associated with several potential optical counterparts. Five months after the initial detection the source was re-detected in $\mathrm{X}$-rays at a flux level nearly ten times lower. This latter detection rules out a GRB or a typical tidal disruption event.

We cannot make a definitive conclusion about the nature of this source. The X-rays could be associated with one of the stars S1 or S2. In this case we have found a very bright and rare stellar flare. Another possible scenario is that the X-rays are emitted by $\mathrm{O} 3$ (or a fainter object undetected in the optical). O3 could either be a distant flaring AGN or it could be a nearby faint star exhibiting a strong X-ray flare.

$\mathrm{X} 6$ is quite faint in X-rays and not detected in gamma rays. We therefore do not consider it a likely source of the detected neutrinos even if it is a flaring AGN.

\section{Appendix B: Observations}

The following tables list the observations and resulting limits by the different telescopes. Table B.1 shows the observations by ASAS-SN and MASTER and Table B.2 shows those by LCO. Table B.3 lists the limits obtained from Swift observations. The limits calculated by VERITAS are shown in Table B.4 and the ones by HAWC in Table B.5. An overview plot including the limits at different wavelengths is shown in Fig. 7. 
Table B.1. Optical observations from MASTER and ASAS-SN.

\begin{tabular}{|c|c|c|c|c|c|}
\hline Telescope & Time, UTC & Time- $t_{0}$ (days) & Filter & Number of exposures and exposure time & $5 \sigma$ limiting mag \\
\hline ASAS-SN Brutus & $2016-01-20.24$ & -28.57 & $V$ & $3(90 \mathrm{~s})$ & 17.5 \\
\hline MASTER-IAC & $2016-01-22$ 22:56:34 & -25.85 & & $3(60 s)$ & 18.5 \\
\hline ASAS-SN Brutus & $2016-01-23.25$ & -25.56 & $V$ & $3(90 \mathrm{~s})$ & 17.1 \\
\hline MASTER-IAC & 2016-01-23 22:14:49 & -24.88 & & $3(60 \mathrm{~s})$ & 18.2 \\
\hline MASTER-IAC & 2016-01-24 23:09:39 & -23.84 & & $3(60 s)$ & 18.1 \\
\hline ASAS-SN Brutus & $2016-01-26.23$ & -22.58 & $V$ & $3(90 s)$ & 17.4 \\
\hline MASTER-Tunka & $2016-01-2713: 12: 46$ & -21.25 & & $3(60 s)$ & 19.1 \\
\hline ASAS-SN Brutus & $2016-01-30.23$ & -18.58 & $V$ & $3(90 s)$ & 17.7 \\
\hline ASAS-SN Brutus & $2016-02-01.22$ & -16.58 & $V$ & $3(90 s)$ & 17.8 \\
\hline ASAS-SN Brutus & $2016-02-03.25$ & -14.56 & $V$ & $3(90 \mathrm{~s})$ & 17.7 \\
\hline MASTER-IAC & 2016-02-14 20:03:58 & -2.97 & & $3(60 s)$ & 18.7 \\
\hline MASTER-Kislovodsk & $2016-02-1517: 56: 50$ & -2.06 & & $6(60 s)$ & 18.7 \\
\hline MASTER-Kislovodsk & $2016-02-18$ 17:15:58 & 0.91 & & $25 \times 2(180 s)$ & $19.4(18.6)$ \\
\hline MASTER-Tunka & 2016-02-18 17:20:21 & 0.92 & & $3(60 s)$ & 17.2 \\
\hline ASAS-SN Brutus & $2016-02-19.22$ & 1.41 & $V$ & $20(90 \mathrm{~s})$ & 18.2 \\
\hline MASTER-Kislovodsk & 2016-02-19 16:37:32 & 1.89 & & $18 \times 2(180 s)$ & $19.2(18.5)$ \\
\hline MASTER-IAC & 2016-02-23 20:11:37 & 6.03 & & $20 \times 2(180 s)$ & $20.7(19.5)$ \\
\hline MASTER-IAC & 2016-02-24 20:32:18 & 7.05 & & $4 \times 2(180 \mathrm{~s})$ & $20.5(19.8)$ \\
\hline MASTER-IAC & 2016-02-25 21:36:18 & 8.09 & & $4 \times 2(180 \mathrm{~s})$ & $20.5(19.7)$ \\
\hline MASTER-Kislovodsk & 2016-02-26 18:49:01 & 8.98 & & $12 \times 2(180 \mathrm{~s})$ & $19.9(19.2)$ \\
\hline MASTER-Kislovodsk & 2016-02-27 16:21:47 & 9.87 & & $20 \times 2(180 s)$ & $20.3(19.9)$ \\
\hline MASTER-IAC & 2016-02-27 22:40:13 & 10.14 & & $3 \times 2(180 \mathrm{~s})$ & $19.4(18.9)$ \\
\hline MASTER-IAC & 2016-02-27 22:59:51 & 10.15 & $B$ & $2(180 \mathrm{~s})$ & $19.0(18.7)$ \\
\hline MASTER-IAC & 2016-02-27 22:59:51 & 10.15 & $I$ & $2(180 \mathrm{~s})$ & 17.0 \\
\hline MASTER-IAC & 2016-02-28 23:08:13 & 11.16 & & $6 \times 2(180 s)$ & 17.8 \\
\hline MASTER-Kislovodsk & 2016-02-29 17:51:45 & 11.94 & & $18 \times 2(180 \mathrm{~s})$ & $20.3(19.8)$ \\
\hline MASTER-IAC & 2016-02-29 20:17:28 & 12.04 & & $4 \times 2(180 \mathrm{~s})$ & $20.4(19.9)$ \\
\hline MASTER-IAC & $2016-02-29$ 20:28:52 & 12.05 & $B$ & $2(180 \mathrm{~s})$ & 20.2 \\
\hline MASTER-IAC & 2016-02-29 20:28:52 & 12.05 & $I$ & $2(180 \mathrm{~s})$ & 18.0 \\
\hline MASTER-Kislovodsk & 2016-03-01 16:31:39 & 12.88 & & $32(180 \mathrm{~s})$ & $20.3(19.9)$ \\
\hline MASTER-IAC & 2016-03-01 21:51:21 & 13.10 & & $4 \times 2(180 \mathrm{~s})$ & $19.9(19.3)$ \\
\hline MASTER-IAC & 2016-03-01 22:14:23 & 13.12 & $B$ & $2(180 \mathrm{~s})$ & 18.8 \\
\hline MASTER-IAC & 2016-03-01 22:14:23 & 13.12 & $I$ & $2(180 \mathrm{~s})$ & 17.2 \\
\hline MASTER-Tunka & 2016-03-02 13:41:01 & 13.76 & & $12(60 s)$ & 18.4 \\
\hline MASTER-Kislovodsk & 2016-03-02 16:40:35 & 13.89 & & $10(180 \mathrm{~s})$ & $19.6(19.0)$ \\
\hline MASTER-Kislovodsk & 2016-03-03 17:04:55 & 14.90 & & $6(180 s)$ & $17.6(17.2)$ \\
\hline MASTER-IAC & 2016-03-03 20:11:40 & 15.03 & & $3 \times 2(180 \mathrm{~s})$ & $20.2(19.7)$ \\
\hline MASTER-IAC & 2016-03-03 20:20:15 & 15.04 & $B$ & $2(180 \mathrm{~s})$ & 19.4 \\
\hline MASTER-IAC & $2016-03-03$ 20:20:15 & 15.04 & $I$ & $2(180 \mathrm{~s})$ & 17.8 \\
\hline MASTER-Kislovodsk & $2016-03-04$ 16:20:27 & 15.87 & & $6(180 s)$ & 18.2 \\
\hline MASTER-IAC & 2016-03-04 20:41:12 & 16.06 & & $12 \times 2(180 s)$ & $20.2(19.3)$ \\
\hline MASTER-Tunka & 2016-03-06 12:24:08 & 17.71 & & $8(60 s)$ & 18.8 \\
\hline MASTER-Tunka & 2016-03-07 12:18:37 & 18.71 & & $12(60-180 \mathrm{~s})$ & $20.0(19.3)$ \\
\hline MASTER-IAC & $2016-03-0721: 44: 32$ & 19.09 & & $3 \times 2(180 s)$ & $19.4(18.7)$ \\
\hline MASTER-Tunka & 2016-03-08 12:17:08 & 19.71 & & $6(180 s)$ & 18.5 \\
\hline MASTER-Kislovodsk & 2016-03-08 17:19:59 & 19.92 & & $6(60 s)$ & 19.1 \\
\hline MASTER-IAC & 2016-03-08 20:15:08 & 20.04 & & $3 \times 2(180 s)$ & $20.3(19.6)$ \\
\hline MASTER-Tunka & 2016-03-09 12:18:41 & 20.71 & & $6(180 \mathrm{~s})$ & $20.0(19.3)$ \\
\hline MASTER-IAC & 2016-03-09 20:13:47 & 21.04 & & $3 \times 2(180 s)$ & $20.2(19.6)$ \\
\hline MASTER-Tunka & 2016-03-10 13:49:52 & 21.77 & & $6(180 s)$ & $19.5(19.0)$ \\
\hline MASTER-Kislovodsk & $2016-03-1017: 57: 18$ & 21.94 & & $10(60 s)$ & 19.1 \\
\hline MASTER-IAC & 2016-03-10 20:16:12 & 22.03 & & $4 \times 2(180 s)$ & $20.3(19.6)$ \\
\hline MASTER-IAC & 2016-03-11 20:11:23 & 23.04 & & $4 \times 2(180 \mathrm{~s})$ & $19.9(19.2)$ \\
\hline MASTER-Tunka & $2016-03-13$ 13:39:33 & 24.76 & & $3(180 \mathrm{~s})$ & 18.8 \\
\hline MASTER-IAC & 2016-03-13 20:18:08 & 25.04 & & $3 \times 2(180 \mathrm{~s})$ & $20.3(19.5)$ \\
\hline MASTER-Tunka & $2016-03-15$ 13:41:19 & 26.76 & & $6(180 \mathrm{~s})$ & $19.0(18.5)$ \\
\hline MASTER-IAC & 2016-03-17 20:31:50 & 29.05 & & $3 \times 2(180 \mathrm{~s})$ & $19.0(18.6)$ \\
\hline MASTER-IAC & 2016-03-18 20:31:42 & 30.05 & & $4 \times 2(180 \mathrm{~s})$ & $19.6(19.0)$ \\
\hline MASTER-IAC & 2016-03-19 20:35:02 & 31.05 & & $3 \times 2(180 s)$ & $19.6(18.7)$ \\
\hline MASTER-IAC & 2016-03-21 20:30:07 & 33.05 & & $3 \times 2(180 s)$ & 18.2 \\
\hline
\end{tabular}

Notes. The columns list the telescope, the start time of the observation, the time relative to the neutrino alert, the band if a filter was used, the number of exposures, the time per exposure and a typical limiting magnitude. A white filter was used for most MASTER observations. The factor $\times 2$ indicates that both tubes of the MASTER twin telescopes pointed at the same position. The limiting magnitudes are for co-added images and the limits for individual images are given in parentheses. All limits correspond to the $5 \sigma$ level. 
Table B.2. Optical observations by LCO.

\begin{tabular}{|c|c|c|c|c|c|c|c|}
\hline $\begin{array}{l}\text { RA } \\
\left({ }^{\circ}\right)\end{array}$ & $\begin{array}{l}\text { Dec } \\
\left({ }^{\circ}\right)\end{array}$ & Obs. date and UTC & $\begin{array}{l}\text { Time }-t_{0} \\
\text { (days) }\end{array}$ & Filter & $\begin{array}{l}\text { Exposure } \\
(\mathrm{s})\end{array}$ & Airmass & $\begin{array}{l}5 \sigma \text { limiting mag } \\
(\mathrm{mag})\end{array}$ \\
\hline 26.46854 & 39.48407 & 2016-02-19 01:53:36 AM & 1.272 & $g$ & 200 & 1.27973 & 21.11 \\
\hline 26.46854 & 39.48411 & 2016-02-19 01:57:54 AM & 1.275 & $r$ & 120 & 1.29248 & 20.58 \\
\hline 25.58188 & 39.48409 & 2016-02-19 02:03:01 AM & 1.279 & $g$ & 200 & 1.32705 & 21.05 \\
\hline 25.58188 & 39.48408 & 2016-02-19 02:07:25 AM & 1.282 & $r$ & 120 & 1.34209 & 20.64 \\
\hline 25.58188 & 39.4841 & 2016-02-19 02:10:40 AM & 1.284 & $i$ & 120 & 1.35507 & 20.31 \\
\hline 26.02522 & 39.4841 & 2016-02-19 02:14:10 AM & 1.287 & $\mathrm{U}$ & 300 & 1.369 & NULL \\
\hline 26.02521 & 39.48409 & 2016-02-19 02:26:07 AM & 1.295 & $B$ & 200 & 1.41944 & 21.04 \\
\hline 26.02521 & 39.48409 & 2016-02-19 02:30:09 AM & 1.298 & $B$ & 200 & 1.43915 & 21.03 \\
\hline 26.02521 & 39.48408 & 2016-02-19 02:34:26 AM & 1.301 & $V$ & 120 & 1.45737 & 20.66 \\
\hline 26.02522 & 39.48409 & 2016-02-19 02:37:13 AM & 1.303 & $V$ & 120 & 1.4718 & 20.72 \\
\hline 26.02521 & 39.48407 & 2016-02-19 02:40:16 AM & 1.305 & $g$ & 200 & 1.49206 & 21.01 \\
\hline 26.02521 & 39.48409 & 2016-02-19 02:44:17 AM & 1.307 & $g$ & 200 & 1.51466 & 20.99 \\
\hline 26.02522 & 39.48409 & 2016-02-19 02:48:31 AM & 1.310 & $r$ & 120 & 1.53538 & 20.46 \\
\hline 26.02521 & 39.48409 & 2016-02-19 02:51:13 AM & 1.312 & $r$ & 120 & 1.55162 & 20.53 \\
\hline 26.02521 & 39.48407 & 2016-02-19 02:54:13 AM & 1.314 & $i$ & 120 & 1.57035 & 20.21 \\
\hline 26.0252 & 39.48408 & 2016-02-19 02:56:57 AM & 1.316 & $i$ & 120 & 1.58775 & 20.14 \\
\hline 26.0252 & 39.04076 & 2016-02-19 03:00:18 AM & 1.319 & $g$ & 200 & 1.61753 & 20.86 \\
\hline 26.02521 & 39.04075 & 2016-02-19 03:04:34 AM & 1.322 & $r$ & 120 & 1.64238 & 20.40 \\
\hline 26.0252 & 39.04074 & 2016-02-19 03:07:37 AM & 1.324 & $i$ & 120 & 1.66441 & 19.91 \\
\hline 26.46856 & 39.4841 & 2016-02-19 03:12:49 AM & 1.327 & $g$ & 200 & 1.69135 & 20.45 \\
\hline 26.46855 & 39.48409 & 2016-02-19 03:17:07 AM & 1.330 & $r$ & 120 & 1.71916 & 20.15 \\
\hline 26.46853 & 39.4841 & 2016-02-19 03:20:52 AM & 1.333 & $i$ & 120 & 1.74817 & 19.47 \\
\hline 26.02521 & 39.48407 & 2016-03-01 02:01:02 AM & 12.277 & $B$ & 200 & 1.51474 & 21.85 \\
\hline 26.0252 & 39.48408 & 2016-03-01 02:05:04 AM & 12.280 & $B$ & 200 & 1.53826 & 21.94 \\
\hline 26.02522 & 39.48413 & 2016-03-01 02:09:23 AM & 12.283 & $V$ & 120 & 1.56018 & 21.52 \\
\hline 26.02521 & 39.48418 & 2016-03-01 02:12:04 AM & 12.285 & $V$ & 120 & 1.57727 & 21.56 \\
\hline 26.0252 & 39.48415 & 2016-03-01 02:15:02 AM & 12.287 & $g$ & 200 & 1.60098 & 22.06 \\
\hline 26.02521 & 39.48412 & 2016-03-01 02:19:03 AM & 12.290 & $g$ & 200 & 1.62804 & 22.29 \\
\hline 26.02522 & 39.48428 & 2016-03-01 02:23:17 AM & 12.293 & $r$ & 120 & 1.65314 & 21.43 \\
\hline 26.02521 & 39.48412 & 2016-03-01 02:25:58 AM & 12.295 & $r$ & 120 & 1.67264 & 21.52 \\
\hline 26.0252 & 39.92745 & 2016-03-03 01:57:31 AM & 14.275 & $B$ & 200 & 1.53699 & 20.49 \\
\hline 26.02522 & 39.92748 & 2016-03-03 02:01:35 AM & 14.278 & $B$ & 200 & 1.56253 & 21.11 \\
\hline 26.02521 & 39.92747 & 2016-03-03 02:06:19 AM & 14.281 & $V$ & 120 & 1.58814 & 21.03 \\
\hline 26.0252 & 39.92742 & 2016-03-03 02:10:00 AM & 14.284 & $V$ & 120 & 1.6115 & 20.71 \\
\hline 26.02522 & 39.92741 & 2016-03-03 02:13:04 AM & 14.286 & $g$ & 200 & 1.63762 & 21.93 \\
\hline 26.0252 & 39.9274 & 2016-03-03 02:17:06 AM & 14.289 & $g$ & 200 & 1.66663 & 21.77 \\
\hline 26.0252 & 39.92746 & 2016-03-03 02:21:22 AM & 14.292 & $r$ & 120 & 1.69315 & 20.60 \\
\hline 26.0252 & 39.9274 & 2016-03-03 02:24:03 AM & 14.293 & $r$ & 120 & 1.71387 & 20.89 \\
\hline 26.0252 & 39.92744 & 2016-03-03 02:26:58 AM & 14.295 & $i$ & 120 & 1.73687 & 20.52 \\
\hline
\end{tabular}

Notes. The limiting magnitudes correspond to the images without running a discovery pipeline and so apply to a source at a known location. The limiting magnitude could not be calculated for the $U$ band because not enough stars are detected in the image to calibrate it.

Table B.3. XRT upper limits.

\begin{tabular}{llll}
\hline \hline $\begin{array}{l}E_{\min } \\
(\mathrm{keV})\end{array}$ & $\begin{array}{l}E_{\max } \\
(\mathrm{keV})\end{array}$ & $\begin{array}{l}\text { Flux upper limit AGN } \\
\left(\mathrm{erg} \mathrm{cm}^{-2} \mathrm{~s}^{-1}\right)\end{array}$ & $\begin{array}{l}\text { Flux upper limit GRB } \\
\left(\mathrm{erg} \mathrm{cm}^{-2} \mathrm{~s}^{-1}\right)\end{array}$ \\
\hline 0.3 & 1 & $(2.67-4.83) \times 10^{-13}$ & $(2.53-4.56) \times 10^{-13}$ \\
1 & 2 & $(2.55-4.61) \times 10^{-13}$ & $(2.58-4.65) \times 10^{-13}$ \\
2 & 10 & $(1.00-1.80) \times 10^{-12}$ & $(0.92-1.67) \times 10^{-12}$ \\
0.3 & 10 & $(6.28-8.92) \times 10^{-13}$ & $(6.56-9.32) \times 10^{-13}$ \\
\hline
\end{tabular}

Notes. All values are in erg $\mathrm{cm}^{-2} \mathrm{~s}^{-1}$ in the specified band. The upper limits are at $3 \sigma$ confidence level.
Table B.4. VERITAS flux upper limits.

\begin{tabular}{lll}
\hline \hline $\begin{array}{l}E_{\min } \\
(\mathrm{TeV})\end{array}$ & $\begin{array}{l}E_{\max } \\
(\mathrm{TeV})\end{array}$ & $\begin{array}{l}\text { Flux upper limit } \\
\left(\mathrm{cm}^{-2} \mathrm{~s}^{-1} \mathrm{TeV}^{-1}\right)\end{array}$ \\
\hline 0.316 & 0.501 & $8.0 \times 10^{-11}$ \\
0.501 & 0.794 & $2.3 \times 10^{-11}$ \\
0.794 & 1.259 & $1.5 \times 10^{-12}$ \\
1.259 & 1.995 & $5.7 \times 10^{-13}$ \\
\hline
\end{tabular}

Notes. Differential flux upper limits for a gamma-ray point-source located at the averaged triplet position. The limits are at $95 \%$ confidence level and do not depend on the spectral shape. 
Table B.5. HAWC flux upper limits.

\begin{tabular}{lllll}
\hline \hline $\begin{array}{l}E_{\min } \\
(\mathrm{TeV})\end{array}$ & $\begin{array}{l}E_{\max } \\
(\mathrm{TeV})\end{array}$ & $\begin{array}{l}\text { Upper limit 1 transit } \\
\left(\mathrm{cm}^{-2} \mathrm{~s}^{-1} \mathrm{TeV}\right)\end{array}$ & $\begin{array}{l}\text { Upper limit 11 transits } \\
\left(\mathrm{cm}^{-2} \mathrm{~s}^{-1} \mathrm{TeV}\right)\end{array}$ & $\begin{array}{l}\text { Upper limit 508 transits } \\
\left(\mathrm{cm}^{-2} \mathrm{~s}^{-1} \mathrm{TeV}\right)\end{array}$ \\
\hline 0.5 & 1.7 & $8.50 \times 10^{-11}$ & $3.86 \times 10^{-11}$ & $3.57 \times 10^{-12}$ \\
1.7 & 5.3 & $3.31 \times 10^{-11}$ & $1.45 \times 10^{-11}$ & $1.03 \times 10^{-12}$ \\
5.3 & 16.7 & $1.45 \times 10^{-11}$ & $6.93 \times 10^{-12}$ & $5.81 \times 10^{-13}$ \\
16.7 & 52.9 & $7.82 \times 10^{-12}$ & $4.68 \times 10^{-12}$ & $2.16 \times 10^{-13}$ \\
52.9 & 167.2 & $6.61 \times 10^{-12}$ & $4.20 \times 10^{-12}$ & $1.15 \times 10^{-13}$ \\
\hline
\end{tabular}

Notes. Flux upper limits at the $95 \%$ confidence level are calculated for the night of the transient during which the neutrino alert was detected (third column), using all data recorded within 14 days after the alert (fourth column) as well as using all recorded data (last column). 